\title{
Single and double coincidence nucleon spectra in the weak decay of $\Lambda$-hypernuclei
}

\author{
E. Bauer ${ }^{1}$, G. Garbarino ${ }^{2}$, A. Parreño ${ }^{3}$ and A. Ramos ${ }^{3}$ \\ ${ }^{1}$ Departamento de Física, Universidad Nacional de La Plata, C. C. 67 e Instituto \\ de Física La Plata, CONICET, 1900 La Plata, Argentina \\ ${ }^{2}$ Dipartimento di Fisica Teorica, Università di Torino and INFN, Sezione di \\ Torino, I-10125 Torino, Italy \\ ${ }^{3}$ Departament d'Estructura i Constituents de la Matèria, Universitat de \\ Barcelona, E-08028 Barcelona, Spain
}

\begin{abstract}
Single and double coincidence nucleon spectra in the weak decay of $\Lambda$ hypernuclei are studied within a nuclear matter formalism extended to finite nuclei via the local density approximation. With respect to previous calculations, the present work adopts a unified microscopic approach for both the one- and two-body induced mechanisms, including the channels $\Lambda n n \rightarrow n n n$ and $\Lambda p p \rightarrow n p p$ in addition to the mode $\Lambda n p \rightarrow n n p$ already considered in earlier phenomenological studies. The propagation of the final nucleons in the residual nucleus is simulated by an intranuclear cascade code. Through the comparison of our predictions with ${ }_{\Lambda}^{12} \mathrm{C}$ KEK nucleon coincidence data obtained with a nucleon kinetic energy threshold of $30 \mathrm{MeV}$ and an opening angle region $\cos \theta_{N N} \leq-0.7$ we determine $\Gamma_{n} / \Gamma_{p}=0.66 \pm 0.24$. We find that the value of $\Gamma_{n} / \Gamma_{p}$ extracted from single nucleon distributions is strongly affected by final state interaction effects. Some discrepancies between measured and calculated proton spectra are also pointed out.
\end{abstract}

Key words: $\Lambda$-Hypernuclei, Non-Mesonic Weak Decay, $\Gamma_{n} / \Gamma_{p}$ ratio, Two-Nucleon Induced Decay

PACS: 21.80.+a, 25.80.Pw

Preprint submitted to Nuclear Physics A

20 January 2010 


\section{Introduction}

Diversified efforts have been devoted to the study of hypernuclear weak decay in the latest years. Theoretical reviews on the subject can be found in [1-3] and recent, related KEK, BNL and FINUDA experiments in [4-17]. New experiments are planned at J-PARC [18] and HypHI [19]. Strong evidences were obtained for a solution of the long standing problem on the ratio $\Gamma_{n} / \Gamma_{p}$ between the widths for the weak non-mesonic processes $\Lambda n \rightarrow n n$ and $\Lambda p \rightarrow n p$. They originated from theoretical analysis [20,21] of KEK two-nucleon coincidence data [8-10]. The problem was mostly due to a non-trivial interpretation of experimental data, which required a careful analysis of nuclear medium effects on the weak decay nucleons, rather than to a poor understanding of the weak decay mechanisms themselves. The lately extracted $\Gamma_{n} / \Gamma_{p}$ values $[20,21]$ turned out to be in agreement with the previous, pure theoretical estimates of [22-28] obtained by using one-meson-exchange potentials to describe the one-nucleon induced, $\Lambda N \rightarrow n N$ weak transitions.

Nevertheless, further theoretical and experimental work is desirable in order to confirm the previously mentioned evidence in favor of a solution of the $\Gamma_{n} / \Gamma_{p}$ puzzle and to have a more accurate determination of the ratio. Indeed, on the one hand such an evidence relies on particular theoretical descriptions of both the weak decay mechanism and the subsequent propagation of the produced nucleons within the residual nucleus. In this direction, the use of alternative weak decay and/or final state interaction models is of interest. On the other hand, one has to consider another problem of the field: it concerns the asymmetry of the protons emitted in the non-mesonic decay of polarized hypernuclei, measured to be not far from zero in recent experiments [29] while a large negative number is predicted by theoretical models [23, 30-32]. In the latest years, a strong effect of nucleon final state interactions (FSI) was pointed out [30], without bringing new hints for a possible solution of the asymmetry puzzle. Recently, however, the dominating effect on the asymmetry of the scalar-isoscalar channel in the weak decay mechanism has been emphasized [33-36], and the two-pion exchange chiral model developed in [22] gives promising results for the rates [22,37] and the asymmetries [37] of both ${ }_{\Lambda}^{5} \mathrm{He}$ and ${ }_{\Lambda}^{12} \mathrm{C}$ hypernuclei. Establishing the connections existing among the weak decay magnitudes: $\Gamma_{n} / \Gamma_{p}, \Gamma_{2}$ (the width for two-nucleon induced decays, $\Lambda N N \rightarrow n N N), \Gamma_{\mathrm{NM}}=\Gamma_{n}+\Gamma_{p}+\Gamma_{2}$ and the asymmetry parameters, and the question concerning the validity of the $\Delta I=1 / 2$ isospin rule in the non-mesonic decay are other important issues which deserve future investigations in the prospect of a better understanding of baryon-baryon weak 
interactions.

On the experimental side, very recent measurements of single- $[4-7,13,15,17]$ and double-coincidence [8-10] nucleon spectra from the non-mesonic hypernuclear decay were reported with improved precision with respect to the ones at disposal in previous experiments [38-42] and in forms that suggest suitable comparisons with theory. Some of these experiments have somehow managed to derive values of $\Gamma_{n} / \Gamma_{p}$. They are discussed in the following paragraphs.

The $\Gamma_{n} / \Gamma_{p}$ ratio was determined by $\mathrm{KEK}-\mathrm{E} 307[4,5]$ for ${ }_{\Lambda}^{12} \mathrm{C},{ }_{\Lambda}^{28} \mathrm{Si}$ and ${ }_{\Lambda} \mathrm{Fe}$ hypernuclei from single-proton kinetic energy spectra measurements and by making use of the intranuclear cascade code of [43] (based on the polarization propagator formalism of [44]) to simulate nucleon re-scattering in the residual nucleus. For ${ }_{\Lambda}^{12} \mathrm{C}, \Gamma_{n} / \Gamma_{p}=0.87 \pm 0.23$ was obtained in [5] by neglecting the two-nucleon induced decay mechanism and $\Gamma_{n} / \Gamma_{p}=0.60_{-0.23}^{+0.25}$ by assuming $\Gamma_{2} /\left(\Gamma_{n}+\Gamma_{p}\right)=\Gamma(\Lambda n p \rightarrow n n p) /\left(\Gamma_{n}+\Gamma_{p}\right)=0.35$. As the very same authors of $[4,5]$ noted in [6], these determinations of the ratio may be affected by the fact that in the experiment the neutron-induced decay width was estimated indirectly, from the proton measurement, using the relation $\Gamma_{n}=\Gamma_{\mathrm{T}}-\Gamma_{p}-\Gamma_{\pi^{-}}-\Gamma_{\pi^{0}}\left(-\Gamma_{2}\right)$. This method also required the measurement of the total decay width $\Gamma_{\mathrm{T}}$ as well as the decay rates for the mesonic channels $\Lambda \rightarrow \pi^{-} p\left(\Gamma_{\pi^{-}}\right)$and $\Lambda \rightarrow \pi^{0} n$ ( $\Gamma_{\pi^{0}}$, for which previous data from [39] were used in the analysis of [5]). Moreover, the severe energy losses suffered by protons inside the (thick) target and detector materials and the consequently high kinetic energy threshold (about $40 \mathrm{MeV}$ ) for proton detection in KEK-E307 did not permit an easy reconstruction of the low energy part of the proton spectrum, which is essential for the indirect evaluation of $\Gamma_{n}$. As a consequence, in [6] the hypothesis was advanced that $\Gamma_{n}\left(\Gamma_{p}\right)$ might be overestimated (underestimated) in the analysis of [5] because of an underestimation in the number of emitted protons.

A controversial determination of $\Gamma_{n} / \Gamma_{p}$ from KEK-E369 data, based on unproven, delicate hypotheses and theoretical input (again from [43]), was reported in [6]. In this experiment, direct measurements of single-neutron kinetic energy spectra were performed (with a $10 \mathrm{MeV}$ threshold) for ${ }_{\Lambda}^{12} \mathrm{C}$ and ${ }_{\Lambda}^{89} \mathrm{Y}$; once analyzed together with the single-proton spectra of $[4,5]$, a ratio $\Gamma_{n} / \Gamma_{p}=0.51 \pm 0.15$ for ${ }_{\Lambda}^{12} \mathrm{C}$ was derived by neglecting the two-nucleon induced decay channel.

To overcome the difficulties of the discussed experiments, both single-neutron and single-proton energy spectra were measured simultaneously by KEK- 
E462 for ${ }_{\Lambda}^{5} \mathrm{He}$ and KEK-E508 for ${ }_{\Lambda}^{12} \mathrm{C}$ [7]. From these measurements, the authors concluded that $\Gamma_{n} / \Gamma_{p} \simeq\left(N_{n} / N_{p}-1\right) / 2 \simeq 0.5$ for both ${ }_{\Lambda}^{5}$ He and ${ }_{\Lambda}^{12} \mathrm{C}$, $N_{n}\left(N_{p}\right)$ being the total number of neutrons (protons) with kinetic energies $T_{N}$ above $60 \mathrm{MeV}$. However, one has to note that the previous approximate relation between $\Gamma_{n} / \Gamma_{p}$ and $N_{n} / N_{p}$ is only valid when FSI and two-nucleon induced decay effects can be neglected. The predictions of [21] and the results presented in Sections 3.1 and 3.2 prove that FSI are not negligible even when a high detection threshold such as $T_{N}^{\text {th }}=60 \mathrm{MeV}$ is used.

In the experiments KEK-E462 $\left({ }_{\Lambda}^{5} \mathrm{He}\right)$ and KEK-E508 $\left({ }_{\Lambda}^{12} \mathrm{C}\right)$, nucleon-nucleon coincidence spectra were also measured [8-10]. Quite clean angular and energy correlations between neutron-neutron and neutron-proton emitted pairs (i.e., back-to-back kinematics and $T_{N_{1}}+T_{N_{2}} \simeq 155 \mathrm{MeV}$ ) were observed, thus representing the first direct experimental evidence of the existence of the onebody induced decays $\Lambda n \rightarrow n n$ and $\Lambda p \rightarrow n p$. The ratio, $N_{n n} / N_{n p}$, between the numbers of emitted neutron-neutron and neutron-proton pairs was measured to be around 0.5 for both ${ }_{\Lambda}^{5} \mathrm{He}$ and ${ }_{\Lambda}^{12} \mathrm{C}$ after applying the angular and energy restrictions: $\cos \theta_{N N} \leq-0.8$ and $T_{N} \geq 30 \mathrm{MeV}$. The authors of [8,9] concluded that, under these constraints, $\Gamma_{n} / \Gamma_{p} \simeq N_{n n} / N_{n p} \simeq 0.5$ on the basis of a supposed cancellation of FSI and two-nucleon stimulated decays effects in the ratio $N_{n n} / N_{n p}$. In a more recent work [10], the result $\Gamma_{n} / \Gamma_{p}=0.51 \pm 0.13 \pm 0.05$ was deduced by the KEK collaboration for ${ }_{\Lambda}^{12} \mathrm{C}$ after correcting for FSI effects by making use of the number of detected proton-proton pairs in addition to measurements of $N_{n n}$ and $N_{n p}$. Two-nucleon stimulated decays were not taken into account. A method similar to the one used in [6] was applied to determine $\Gamma_{n} / \Gamma_{p}$. We shall widely discuss the non-negligible effect of FSI in the extraction of $\Gamma_{n} / \Gamma_{p}$ from measurements of $N_{n n} / N_{n p}$ in Section 3.5.

Despite this recent experimental progress, improved and/or independent measurements are awaited for a really complete understanding of the $\Lambda N \rightarrow n N$ reaction in nuclei. On this respect, the observation of the weak decay of neutron and proton rich hypernuclei $[14,19]$ would also be source of new information. Beyond these considerations, from the above paragraphs it is clear that the extraction of the $\Gamma_{n} / \Gamma_{p}$ ratio from data is a complex task, once FSI and the two-body induced mechanism play non-negligible roles. A theoretical approach for the extraction of $\Gamma_{n} / \Gamma_{p}$ from experimental spectra (which, contrary to the decay rates, are the actual observable quantities) is required. Values of $\Gamma_{n} / \Gamma_{p}$ determined in such a way are thus model-dependent; only when different approaches lead to the same $\Gamma_{n} / \Gamma_{p}$ it is fair to call this ratio the experimental value of $\Gamma_{n} / \Gamma_{p}$. 
On the theoretical side, an extensive study of single- and double-coincidence nucleon spectra for the non-mesonic decay of ${ }_{\Lambda}^{5} \mathrm{He}$ and ${ }_{\Lambda}^{12} \mathrm{C}$ hypernuclei was presented in [20,21]. A one-meson-exchange (OME) potential was used for the $\Lambda N \rightarrow n N$ transition in a finite nucleus framework. The model adopted for the two-nucleon induced decay channel is described in [44]. In simple terms, this model results from the product of an approximation to the $3 p 2 h$ phase space times a constant which embodies, phenomenologically, the strong interaction dynamics. The process was assumed to proceed entirely from the $\Lambda n p \rightarrow n n p$ reaction. The calculation was performed in nuclear matter together with the local density approximation. The intranuclear cascade code of [43] was used to simulate the nucleon propagation inside the residual nucleus. Comparison with KEK-E462 and KEK-E508 two-nucleon coincidence data [8,9] lead to the determination of $\Gamma_{n} / \Gamma_{p}$ values around 0.3-0.4 for both ${ }_{\Lambda}^{5} \mathrm{He}$ and ${ }_{\Lambda}^{12} \mathrm{C}[21]$. It was shown that FSI and two-nucleon induced decays affect the extraction of $\Gamma_{n} / \Gamma_{p}$ from two-nucleon coincidence data even when favorable energy and angular correlation restrictions are imposed on the observed nucleon pairs. It should be noted that an asymmetric treatment was given to one- and twobody induced decay mechanisms in [20,21]: while the one-body stimulated decay is evaluated in a finite nucleus framework, an approximated model in nuclear matter is used in the calculation of the two-body induced decay.

The rather simple two-body induced decay model employed in those works, together with the non-negligible role played by the two-body stimulated process in the interpretation of data, have motivated us to explore a unified approach for both the one- and the two-body stimulated decay mechanisms. Our scheme for the decay rates, developed in [25-28], puts on the same ground the microscopic evaluation of one- and two-body stimulated decay mechanisms and includes the channels $\Lambda n n \rightarrow n n n$ and $\Lambda p p \rightarrow n p p$ besides the standard mode $\Lambda n p \rightarrow n n p$. This scheme uses the same OME weak transition potential (containing $\pi, \rho, K, K^{*}, \omega$ and $\eta$ exchange) of [23]. The huge number of two-body induced configurations makes the use of nuclear matter more convenient, the predictions being extended to finite nuclei via the local density approximation. Most of the results shown in the present work are for the intermediate-mass hypernucleus ${ }_{\Lambda}^{12} \mathrm{C}$, but we also present some results for a heavier hypernucleus, ${ }_{\Lambda}^{89}$ Y. FSI are evaluated by means of the Monte Carlo intranuclear cascade code of [43]. Our aim is twofold. First, we want to discuss predictions for the nucleon emission spectra using the unified microscopic approach. Afterwards, by fitting experimental spectra we address the question of the determination of the experimental value of $\Gamma_{n} / \Gamma_{p}$. Our main achievement is an agreement of the present results with the ones of $[20,21]$ concerning the ratio $N_{n n} / N_{n p}$. This supports the idea that it is possible to determine from data a basically model 
independent value for $\Gamma_{n} / \Gamma_{p}$, which then truly qualifies as the experimental value of the ratio.

The paper is organized in the following way. The weak decay model employed in the calculation is outlined in Section 2.1. In Section 2.2 we give a very brief summary of the intranuclear cascade simulation. Numerical results for single and double coincidence nucleon distributions are presented and compared with data in Section 3. The contribution of the two-nucleon induced decay channels is analyzed with special regard in this Section. Finally, our conclusions are drawn in Section 4.

\section{Model}

\subsection{Weak decay}

Let us consider the one and two-nucleon induced non-mesonic weak decay widths for a $\Lambda$-hyperon with four-momentum $k=\left(k_{0}, \boldsymbol{k}\right)$ inside infinite nuclear matter with Fermi momentum $k_{F}$. In a schematic way, one can write:

$$
\Gamma_{1(2)}\left(k, k_{F}\right)=\sum_{f}\left|\left\langle f\left|V^{\Lambda N \rightarrow N N}\right| 0\right\rangle_{k_{F}}\right|^{2} \delta\left(E_{f}-E_{0}\right)
$$

where $V^{\Lambda N \rightarrow N N}$ is the weak transition potential, $|0\rangle_{k_{F}}$ denotes the initial hypernuclear ground state with energy $E_{0}$ and $|f\rangle$ stands for a possible $2 p 1 h$ or $3 p 2 h$ final state with energy $E_{f}$. The $2 p 1 h$ and $3 p 2 h$ final states contribute to $\Gamma_{1}$ and $\Gamma_{2}$, respectively.

The decay rates for a finite hypernucleus are obtained via the local density approximation [45]

$$
\Gamma_{1(2)}=\int d \boldsymbol{k}\left|\widetilde{\psi}_{\Lambda}(\boldsymbol{k})\right|^{2} \int d \boldsymbol{r}\left|\psi_{\Lambda}(\boldsymbol{r})\right|^{2} \Gamma_{1(2)}\left(\boldsymbol{k}, k_{F}(r)\right)
$$

i.e., by averaging the partial width over the nuclear volume, assuming a local Fermi momentum $k_{F}(r)=\left\{3 \pi^{2} \rho(r) / 2\right\}^{1 / 3}$, where $\rho(r)$ is the density profile of the nuclear core. This average is weighted by the probability per unit volume of finding the $\Lambda$ at a given position $\boldsymbol{r},\left|\psi_{\Lambda}(\boldsymbol{r})\right|^{2}$. In a hypernucleus, one needs to further average over the extended momentum distribution of the $\Lambda$ hyperon, $\widetilde{\psi}_{\Lambda}(\boldsymbol{k})$, the Fourier transform of $\psi_{\Lambda}(\boldsymbol{r})$, for which we adopt a $1 s_{1 / 2}$ harmonic oscillator wave-function with frequency $\hbar \omega=10.8 \mathrm{MeV}$ adjusted to 
the experimental energy separation between the $s$ and $p \Lambda$-levels in ${ }_{\Lambda}^{12} \mathrm{C}$. The $\Lambda$ hyperon energy is given by $k_{0}=m_{\Lambda}+\boldsymbol{k}^{2} /\left(2 m_{\Lambda}\right)+V_{\Lambda}$, i.e., it also contains an experimental binding term $V_{\Lambda}=-10.8 \mathrm{MeV}$.

Since $V^{\Lambda N \rightarrow N N}$ is a two-body operator, the emission of two nucleons may originate either from the Hartree-Fock vacuum or from ground state correlations induced by the nucleon-nucleon interaction. At variance, the emission of three nucleons can only be achieved when $V^{\Lambda N \rightarrow N N}$ acts over a ground state correlation. It is therefore convenient to introduce the following model for the hypernuclear ground state wave-function [27]:

$$
|0\rangle_{k_{F}}=\mathcal{N}\left(k_{F}\right)\left(|\rangle-\sum_{p_{2}^{\prime} h_{2} p_{3} h_{3}} \frac{\left\langle p_{2}^{\prime} h_{2} p_{3} h_{3}\left|V^{N N}\right|\right\rangle}{\varepsilon_{p_{2}^{\prime}}-\varepsilon_{h_{2}}+\varepsilon_{p_{3}}-\varepsilon_{h_{3}}}\left|p_{2}^{\prime} h_{2} p_{3} h_{3}\right\rangle\right) \otimes\left|p_{\Lambda}\right\rangle
$$

where |\rangle is the uncorrelated ground state wave-function, i.e., the HartreeFock vacuum, while the second term in the rhs represents $2 p 2 h$ correlations introduced by the nuclear residual interaction $V^{N N}$. The particular labeling $p_{2}^{\prime}, h_{2}, p_{3}$ and $h_{3}$ (each symbol denoting particle and hole four-momenta and spin and isospin projections) is explained in Subsec. 2.1.2. Besides, $\left|p_{\Lambda}\right\rangle$ is the normalized state of the $\Lambda$, the particle and hole energies are denoted by $\varepsilon_{i}$ and:

$$
\mathcal{N}\left(k_{F}\right)=\left(1+\sum_{p_{2}^{\prime} h_{2} p_{3} h_{3}}\left|\frac{\left\langle p_{2}^{\prime} h_{2} p_{3} h_{3}\left|V^{N N}\right|\right\rangle}{\varepsilon_{p_{2}^{\prime}}-\varepsilon_{h_{2}}+\varepsilon_{p_{3}}-\varepsilon_{h_{3}}}\right|^{2}\right)^{-1 / 2}
$$

is the ground state normalization factor. The explicit expression for $\mathcal{N}\left(k_{F}\right)$ is given in [28].

\subsubsection{One-nucleon induced decay}

By inserting Eq. (3) into Eq. (1), one obtains for $\Gamma_{1}$ :

$$
\Gamma_{1}\left(\boldsymbol{k}, k_{F}\right)=\mathcal{N}^{2}\left(k_{F}\right) \sum_{f}\left|\left\langle f\left|V^{\Lambda N \rightarrow N N}\right| p_{\Lambda}\right\rangle\right|^{2} \delta\left(E_{f}-E_{0}\right)
$$

the final states $|f\rangle$ being restricted to $2 p 1 h$ states. Note that in this expression we have kept only the dominant Hartree-Fock vacuum contribution. The corresponding Goldstone diagrams for the direct and exchange $\Lambda$ self-energies are shown in Fig. 1. Explicit expressions for $\Gamma_{1}$ are found in [25]. 


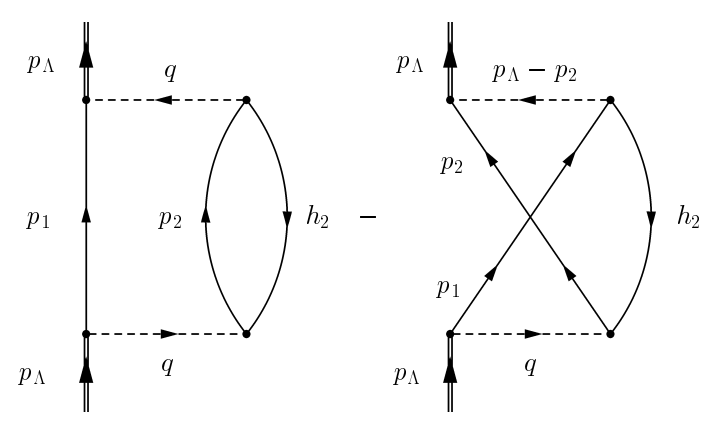

Fig. 1. Direct and exchange $\Lambda$ self-energy diagrams corresponding to the one-nucleon induced decay channel in nuclear matter.

\subsubsection{Two-nucleon induced decay}

We consider now the two-nucleon induced decay mode. From Eqs. (1) and (3), one gets for $\Gamma_{2}$ :

$$
\begin{aligned}
\Gamma_{2}\left(\boldsymbol{k}, k_{F}\right)= & \mathcal{N}^{2}\left(k_{F}\right) \sum_{f} \mid \sum_{p_{2}^{\prime} h_{2} p_{3} h_{3}}\left\langle f\left|V^{\Lambda N \rightarrow N N}\right| p_{2}^{\prime} h_{2} p_{3} h_{3} ; p_{\Lambda}\right\rangle \\
& \times\left.\frac{\left\langle p_{2}^{\prime} h_{2} p_{3} h_{3} ; p_{\Lambda}\left|V^{N N}\right| p_{\Lambda}\right\rangle}{\varepsilon_{p_{2}^{\prime}}-\varepsilon_{h_{2}}+\varepsilon_{p_{3}}-\varepsilon_{h_{3}}}\right|^{2} \delta\left(E_{f}-E_{0}\right),
\end{aligned}
$$

where $|f\rangle$ stands now for the possible $3 p 2 h$ states. At variance with $\Gamma_{1}$, several Goldstone diagrams contribute to $\Gamma_{2}$. A microscopic evaluation of the twonucleon induced decay is a quite involved task which should be pursued on a step-by-step basis.

The complexity of the problem makes the phenomenological approaches [44, 46] very valuable because they were able to establish a first estimation of the size of the two-body induced decay mechanism, as compared to the dominant one-nucleon induced one, as well as its effect on the determination of the ratio $\Gamma_{n} / \Gamma_{p}$. In fact the first model for evaluating $\Gamma_{2}$ [46] was proposed in the hope of understanding the large fraction of neutrons, and hence the large value of $\Gamma_{n} / \Gamma_{p}$, observed in the experiments. The work was inspired by the previous knowledge on pion absorption. The idea was that the near onshell pion produced at the weak vertex would be preferentially absorbed by a neutron-proton pair producing twice as many neutrons than protons from this process. Thereafter, a hybrid model was employed in [44], where the strength of the mechanism was extracted from the phenomenology of the two-nucleon absorption of real pions, and the extension to other kinematical regions was done by taking an approximation for the $2 p 2 h$ phase space. It was explicitly found that the consideration of the two-nucleon induced channel has a 
non-negligible effect on the extraction of the ratio $\Gamma_{n} / \Gamma_{p}$ from the number of emitted protons and neutrons. A following work [43] determined the energy distribution of nucleons, in order see whether the two decay mechanisms, one- and two-nucleon induced, could be disentangled for a proper evaluation of $\Gamma_{n} / \Gamma_{p}$. These previous studies, based on a phenomenological evaluation of $\Gamma_{2}$, established the basis for the analyses performed in [20,21] of experimental two-nucleon coincidence nucleon spectra $[8,9]$, which contributed to the solution of the so-called $\Gamma_{n} / \Gamma_{p}$ puzzle. It is therefore important to check whether the value of the ratio is stable against the use of different models of $\Gamma_{2}$, in other words, to try to establish a model-independent value for $\Gamma_{n} / \Gamma_{p}$. This is the main purpose of this work. In particular, we will employ a model which is based on the same microscopic approach as that adopted for the calculation of the one-nucleon induced mechanisms. Our unified scheme considers a full meson-exchange model in the weak transition potential and $2 p 2 h$ intermediate states excited by a realistic strong $N N$ interaction. This produces a quite different energy distribution of the three nucleons emitted in the two-nucleon absorption processes, as we will see in Sect. 3.4.

In the present work we will keep only the dominating terms of the two-nucleon stimulated contributions, namely those obtained by attaching the weak transition potential $V^{\Lambda N \rightarrow N N}$ to the same $1 p 1 h$ bubble, an example of which is seen in Fig. 2. Actually, a more complete set of diagrams, including terms with the two weak transition potentials connected to different $1 p 1 h$ bubbles as well as Pauli exchange contributions, were discussed in $[27,28]$. The implementation of the full set of diagrams in the evaluation of the spectra is quite involve and goes beyond the scope of the present contribution. Moreover, by keeping the dominating terms it is expected that these missing terms would not modify the value extracted for $\Gamma_{n} / \Gamma_{p}$ in a significant way. Therefore the conclusions of the present work should not be altered. Note that there are three different contributions to the dominating terms, which we denote by $\Gamma_{2}^{\mathrm{pp}}, \Gamma_{2}^{\mathrm{ph}}$ and $\Gamma_{2}^{\mathrm{hh}}\left(\Gamma_{2}=\Gamma_{2}^{\mathrm{pp}}+\Gamma_{2}^{\mathrm{ph}}+\Gamma_{2}^{\mathrm{hh}}\right)$. In the first one, the two $V^{\Lambda N \rightarrow n N}$ are attached to the same particle (see Fig. 2, from which the notation employed in Eqs. (3) and (4) becomes clear). In the ph contribution one $V^{\Lambda N \rightarrow n N}$ is connected to a particle and the other one to a hole. Finally, the two potentials are attached to the same hole for the hh part. The corresponding ph and hh Goldstone diagrams can be found in [26] (Fig. 1) together with their analytical expressions. In addition, the isospin summation in Eq. (6) allows three isospin decay channels, $\Gamma_{2}=\Gamma_{n n}+\Gamma_{n p}+\Gamma_{p p}$, with: $\Gamma_{n n} \equiv \Gamma(\Lambda n n \rightarrow n n n)$, $\Gamma_{n p} \equiv \Gamma(\Lambda n p \rightarrow n n p)$ and $\Gamma_{p p} \equiv \Gamma(\Lambda p p \rightarrow n p p)$. The results obtained in [26] imply $\Gamma_{n p}: \Gamma_{p p}: \Gamma_{n n} \approx 0.78: 0.17: 0.05$, which points to the need of incorporating also the effect of the isospin contributions $\Gamma_{p p}$ and $\Gamma_{n n}$, neglected in 


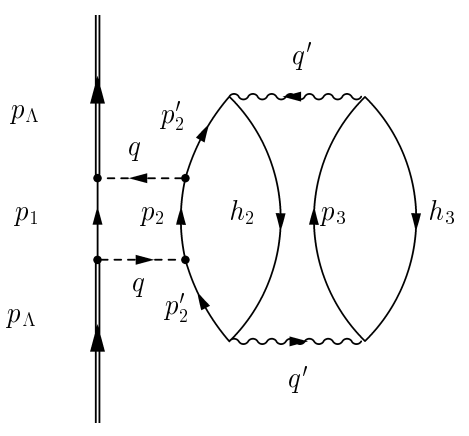

Fig. 2. pp-part of the two particle-two hole contribution to the $\Lambda$ self-energy in nuclear matter.

the phenomenological models both for the evaluation of the nucleon spectra and for the extraction of the $\Gamma_{n} / \Gamma_{p}$ ratio from data. Finally, we point out that for the nuclear residual interaction $V^{N N}$ (which enters both $\Gamma_{2}$ and $\mathcal{N}\left(k_{F}\right)$ ) we have used the Bonn potential [47] in the framework of the parametrization presented in [48], which contains the exchange of $\pi, \rho, \sigma$ and $\omega$ mesons.

\subsection{Intranuclear cascade}

After the $\Lambda$ interacts with one (two) nucleons, the two (three) produced nucleons will interact with other nucleons in their way out of the nucleus. This process, which will generate secondary nucleons, is accounted for by the intranuclear cascade model described in [43].

This model considers a semiclassical propagation of primary (i.e., weak decay) and secondary nucleons. Nucleons move along classical, straight trajectories between collision points and under the influence of a local (i.e., $r$-dependent) mean potential, $V(r)=-k_{F}(r)^{2} / 2 m$, where $k_{F}(r)=\left[3 \pi^{2} \rho(r) / 2\right]^{1 / 3}$ is the local nucleon Fermi momentum corresponding to the nucleon density $\rho(r)$. Propagating nucleons collide with the nucleons of the medium according to free-space nucleon-nucleon cross sections properly corrected to take into account the Pauli blocking and Fermi motion effects. At small energies, for which the nucleon wavelength is comparable in size to the average distance between nucleons, a semiclassical nucleon propagation is not appropriate. For this reason we do not consider our spectra below $30 \mathrm{MeV}$ kinetic energy to be realistic. A quantum-mechanical simulation of FSI would be preferred but it is not yet available. We finally note that the adopted code was tested successfully in a variety of other reactions [49]. For more details of the code we refer to [43]. 


\section{Results}

We start our discussion by observing that the models of $[23,25,26]$ on which the present work is based have been recently improved. On one hand, a more realistic $\Lambda$ wave-function, obtained in terms of the experimental hyperon binding energy, has been considered in the local density approximation (LDA) calculation of $[25,26]$, leading to a reduction of about $25 \%$ on the one-nucleon induced (hereafter referred to as $1 N$-induced) decay rates, $\Gamma_{n}$ and $\Gamma_{p}$, and of $30-40 \%$ on the two-nucleon induced ( $2 N$-induced) ones, $\Gamma_{n n}, \Gamma_{n p}$ and $\Gamma_{p p}$, with respect to the original results. In addition, the normalization of the hypernuclear ground state (see Eqs. (3) and (4)) produces a further reduction of both $\Gamma_{1}$ and $\Gamma_{2}$ of about $30 \%$. The finite nucleus approach of [23] has been improved by using numerically more accurate distorted final state wave-functions and the rates increase by about $15 \%$. In both cases the ratio $\Gamma_{n} / \Gamma_{p}$ is essentially left unchanged. Fortunately, being normalized per non-mesonic weak decay, the spectra discussed in [20,21] are not affected by the corrections of [23].

The updated predictions, used in the present work, are given in Table 1 together with experimental results for $\Gamma_{n}, \Gamma_{p}, \Gamma_{\mathrm{NM}}, \Gamma_{n} / \Gamma_{p}$ and $\Gamma_{2} / \Gamma_{\mathrm{NM}}$. The two sets of results for the finite nucleus calculation, denoted by OMEa and OMEf, are obtained by using, respectively, the Nijmegen potentials NSC97a and NSC97b [50] for the strong vertices and final state $N N$ interactions. The weak transition potential $V^{\Lambda N \rightarrow N N}$ is modeled, in both finite nucleus and LDA models, by the exchange of the $\pi, \eta, K, \rho, \omega$ and $K^{*}$ mesons, whose formulation has been taken from [23]. The finite nucleus results for $\Gamma_{\mathrm{NM}}$ underestimate data, as expected, since they lack the contribution of the $2 N$-stimulated decay mechanism. However, the results obtained for $\Gamma_{n}$ and $\Gamma_{p}$ within the model OMEf are in good agreement with the data of [12]. The LDA prediction for $\Gamma_{\mathrm{NM}}$ slightly underestimates the most recent data of $[8,12]$ but agrees with the previous determinations of $[5,41]$.

Concerning $\Gamma_{n} / \Gamma_{p}$, with the exception of the data from [12], we have only quoted in Table 1 experimental determinations obtained from single-nucleon measurements. Most of these determinations appear to be underestimated by any theoretical prediction found in the literature, which leads to the well known $\Gamma_{n} / \Gamma_{p}$ puzzle. We note that large experimental errors affect all but the most recent data, as mentioned in the Introduction. On the contrary, the analyses of recent two-nucleon coincidence data performed in [21] enabled to determine central values for $\Gamma_{n} / \Gamma_{p}$ around $0.3-0.4$, in agreement with pure theoretical estimates. 
Table 1

Non-mesonic weak decay rates (in units of the free $\Lambda$ decay width) predicted for ${ }_{\Lambda}^{12} \mathrm{C}$ by the updated finite nucleus approach (OMEa and OMEf calculations) of [23] and LDA model of [25-27].

\begin{tabular}{|c|c|c|c|c|c|c|c|}
\hline & $\Gamma_{n}$ & $\Gamma_{p}$ & $\Gamma_{n n}$ & $\Gamma_{n p}$ & $\Gamma_{p p}$ & $\Gamma_{n} / \Gamma_{p}$ & $\Gamma_{\mathrm{NM}}$ \\
\hline $\mathrm{FNa}$ & 0.190 & 0.625 & & & & 0.303 & 0.815 \\
\hline FNf & 0.173 & 0.484 & & & & 0.356 & 0.657 \\
\hline \multirow[t]{2}{*}{ LDA } & 0.155 & 0.543 & 0.010 & 0.138 & 0.036 & 0.285 & 0.882 \\
\hline & $\Gamma_{n}$ & $\Gamma_{p}$ & & $\Gamma_{2} / \Gamma_{\mathrm{NM}}$ & & $\Gamma_{n} / \Gamma_{p}$ & $\Gamma_{\mathrm{NM}}$ \\
\hline FINUDA [17] & & & & $0.24 \pm 0.10$ & & & \\
\hline KEK-E508 [12] & $0.23 \pm 0.08$ & $0.45 \pm 0.10$ & & $0.29 \pm 0.13$ & & $0.51 \pm 0.14$ & $0.95 \pm 0.04$ \\
\hline KEK-E508 [8] & & & & & & & $0.953 \pm 0.032$ \\
\hline KEK-E369 [6] & & & & & & $0.51 \pm 0.15$ & \\
\hline KEK-E307 [5] & & & & & & $0.87 \pm 0.09 \pm 0.21$ & $0.828 \pm 0.056 \pm 0.066$ \\
\hline KEK [41] & & & & & & $1.87 \pm 0.59_{-1.00}^{+0.32}$ & $0.89 \pm 0.15 \pm 0.03$ \\
\hline BNL [40] & & & & & & $1.33_{-0.81}^{+1.12}$ & $1.14 \pm 0.20$ \\
\hline $\mathrm{BNL}[38]$ & & & & & & $0.58 \pm 0.18$ & \\
\hline
\end{tabular}

Concerning the $2 N$-stimulated decay widths, the dominant contribution (about $75 \%$ ) of the microscopic calculation originates from the $n p$-induced decay, $\Lambda n p \rightarrow n n p$. Nevertheless, the other $2 N$-induced channels cannot be neglected. The LDA prediction $\Gamma_{2} / \Gamma_{\mathrm{NM}}=0.21$ is in agreement with the phenomenological estimate of $[20,21], \Gamma_{2} / \Gamma_{\mathrm{NM}}=0.20$, as well as with the very recent experimental determinations of the FINUDA collaboration [17] and of KEK-E508 [12] reported in Table 1.

\subsection{Single-nucleon spectra}

In Figure 3 we compare results of the present LDA calculation with those of the previous finite nucleus evaluation of $[20,21]$. The number of primary protons emitted in the $1 N$-induced non-mesonic weak decay of ${ }_{\Lambda}^{12} \mathrm{C}$ is given as a function of the proton kinetic energy. In order to make the comparison model independent, the spectra are normalized per $1 N$-induced decay assuming $\Gamma_{n} / \Gamma_{p}=\left(\Gamma_{n} / \Gamma_{p}\right)^{\mathrm{LDA}}=0.285$, which is equivalent to normalize per the corresponding proton-induced decay rate. We observe that the Full Width at Half Maximum (FWHM) of the LDA distribution is 15-20 MeV larger than the one of the finite nucleus spectrum, which should be considered more realistic. This is essentially due to the larger dispersion of Fermi motion in the nuclear matter calculation.

In Figure 4 we show the single-proton kinetic energy spectra for the nonmesonic weak decay of ${ }_{\Lambda}^{12} \mathrm{C}$ once $2 N$-induced decays and FSI effects are in- 


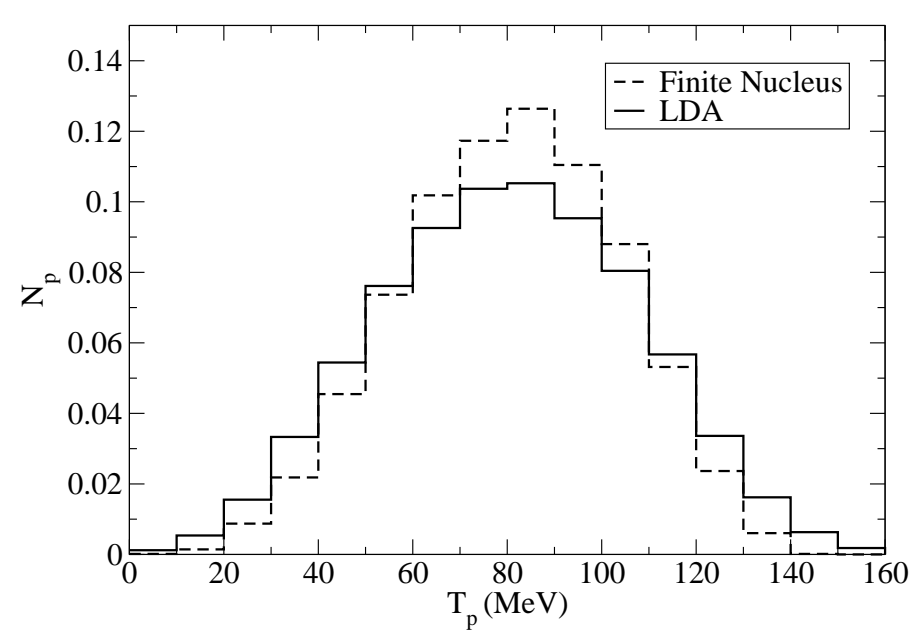

Fig. 3. Kinetic energy spectra of primary protons from the $1 N$-induced non-mesonic weak decay of ${ }_{\Lambda}^{12} \mathrm{C}$. The continuous line refers to the present LDA calculation, the dashed one to the finite nucleus evaluation of $[20,21]$. Both curves are normalized per $1 N$-induced decay assuming $\Gamma_{n} / \Gamma_{p}=\left(\Gamma_{n} / \Gamma_{p}\right)^{\mathrm{LDA}}=0.285$.

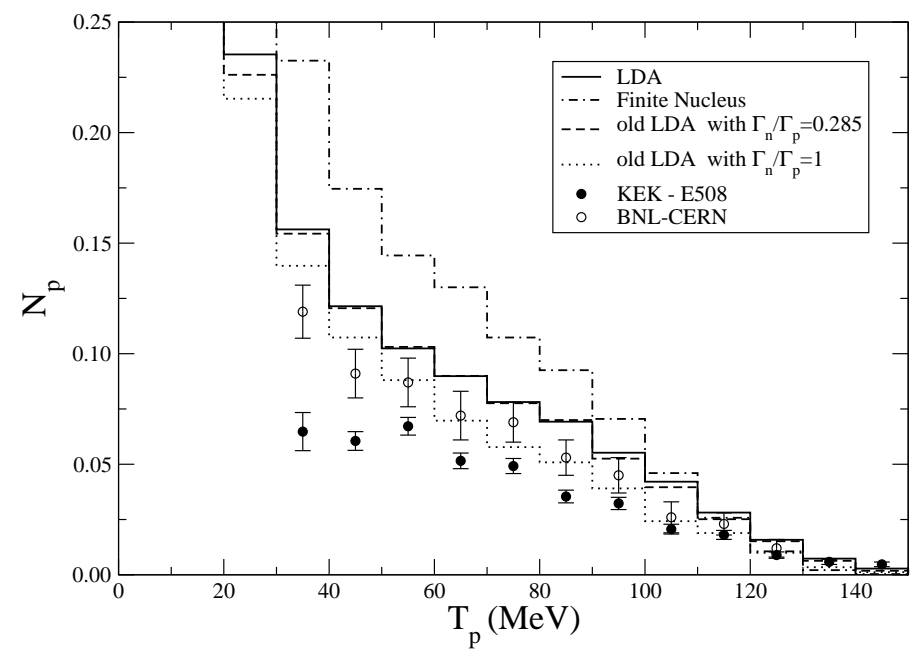

Fig. 4. Single-proton kinetic energy spectra for the non-mesonic weak decay of ${ }_{\Lambda}^{12} \mathrm{C}$ after $2 N$-induced decays and FSI effects are included. Data are from KEK-E508 [7] and Montwill et al. [38]. All results are normalized per non-mesonic weak decay. See detailed explanation in the text.

cluded. All spectra are normalized per non-mesonic weak decay. The continuous line refers to the present LDA result. The dashed line has been obtained with the LDA of [43], where the weak transition potential was described by a correlated pion-exchange. The old LDA spectrum has been obtained assuming same $\Gamma_{n} / \Gamma_{p}$ of the present model, $\Gamma_{n} / \Gamma_{p}=\left(\Gamma_{n} / \Gamma_{p}\right)^{\mathrm{LDA}}=0.285$. Good agreement is found between the two LDA calculations despite the different weak decay models employed. The dot-dashed line has been taken from the finite nucleus evaluation of $[20,21]$ fixing $\Gamma_{n} / \Gamma_{p}=\left(\Gamma_{n} / \Gamma_{p}\right)^{\mathrm{LDA}}=0.285$. Again, the 
differences between LDA and finite nucleus estimates are mainly due to the different phase spaces in the two cases.

As it is apparent from Figure 4, all the theoretical spectra are in strong disagreement with KEK-E508 data [7]. The LDA results are closer to the data by Montwill et al. [38 $]^{1}$, especially if we a large value of $\Gamma_{n} / \Gamma_{p}$ such as 1 is enforced in the old LDA (dotted curve). We recall that such LDA model [43] was also able to reproduce the KEK-E307 single-proton data of [5] with values of $\Gamma_{n} / \Gamma_{p}$ larger than 0.6 , as reported in the Introduction. Note, however, that the proton spectrum obtained using the more realistic phase space of the finite nucleus model (dot-dashed line) is in disagreement with all experimental single-proton spectra. Apparently, given the smooth dependence of the normalized proton yield on $\Gamma_{n} / \Gamma_{p}$, the only way to reconcile the theoretical predictions of models having $\Gamma_{n} / \Gamma_{p} \leq 1$ with the experimental data would be the assumption of substantial modifications in the FSI approach employed, but this would then spoil the success found by the same approach in other nuclear reactions [49]. Note also that there exist in the literature two other experiments giving the distribution of protons following the weak decay of ${ }_{\Lambda}^{12} \mathrm{C}$, that of Ref. [40] and the recent one from the FINUDA collaboration [15, 17]. Since these spectra are unnormalized they cannot be displayed directly in Fig. 4. However, there is a noticeable qualitative difference: the shape of the recent FINUDA spectrum shows a shallow peak at $T_{p} \simeq 80 \mathrm{MeV}$ (the back-to-back kinematics) that no theoretical calculation obtains and which is also lacking in any of the other experimental data sets. This is an interesting point that deserves a more careful study. Given the present dispersion of the different experimental results (not only in shape but also in size), we cannot yet use this observable to constrain the various non-mesonic weak decay models.

In Fig. 5 the results of the present LDA calculation for the the single-neutron spectrum are shown, both for the primary neutrons coming from the $1 N-$ induced mechanism (dashed line) and after including $2 N$-induced decays and FSI effects (solid line). The full result is in good agreement with KEK-E369 data but overestimates somewhat the more precise KEK-E508 spectrum. Note also that a more realistic finite nucleus calculation, based on the same onemeson-exchange model for the weak transition, also obtains agreement with the KEK-E369 data (see Fig. 6 of Ref. [21]), but going through the higher

1 Actually, Montwill's emulsion data refer to a a mixture of boron $\left({ }_{\Lambda}^{9} \mathrm{~B},{ }_{\Lambda}^{10} \mathrm{~B}\right.$ and $\left.{ }_{\Lambda}^{11} \mathrm{~B}\right)$, carbon $\left({ }_{\Lambda}^{11} \mathrm{C},{ }_{\Lambda}^{12} \mathrm{C}\right.$ and $\left.{ }_{\Lambda}^{13} \mathrm{C}\right)$ and nitrogen $\left({ }_{\Lambda}^{13} \mathrm{~N},{ }_{\Lambda}^{14} \mathrm{~N}\right.$ and $\left.{ }_{\Lambda}^{15} \mathrm{~N}\right)$ hypernuclei. As expected, the proton spectra we predict for all these hypernuclei are very similar to each other. It is thus justified to compare Montwill's spectrum with other data and our results for ${ }_{\Lambda}^{12} \mathrm{C}$, as done in Figure 4 . 


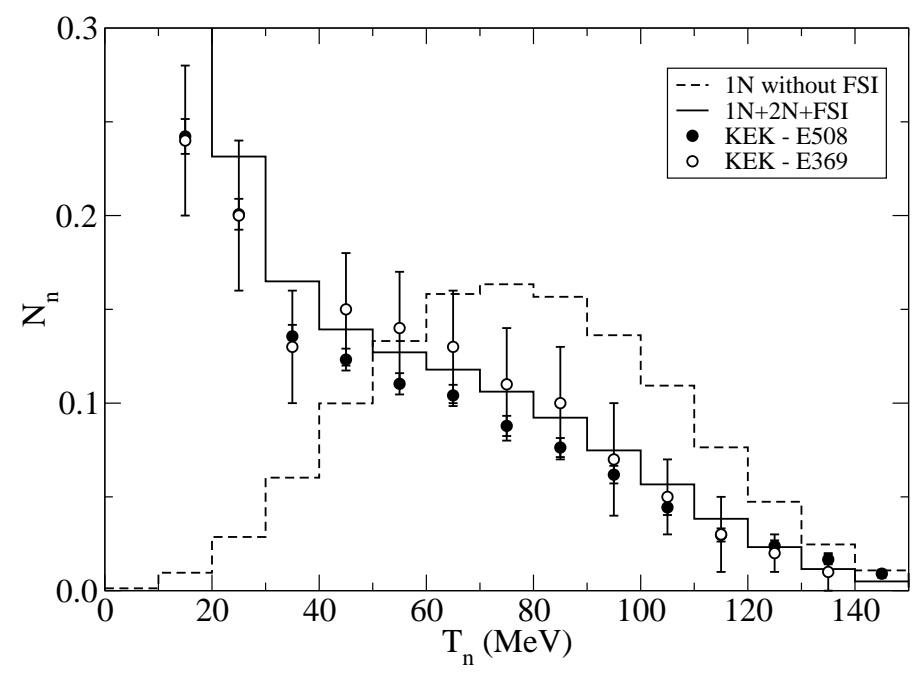

Fig. 5. Single-neutron kinetic energy spectra for the non-mesonic decay of ${ }_{\Lambda}^{12} \mathrm{C}$, obtained from our LDA model which predicts $\Gamma_{n} / \Gamma_{p}=0.285$. The dashed line corresponds to the distribution of primary neutrons, while the continuous line is obtained once $2 N$-induced decays and FSI effects are included. Data are from KEK-E369 [6] and KEK-E508 [7]. The spectrum of primary neutrons (experimental data and the full theoretical result) is (are) normalized per $1 N$-induced (total) non-mesonic weak decay.

side of the error bars.

The evolution of the neutron spectrum with the nuclear size can be easily explored in a LDA-type approach, whereas it would become a prohibitive task in a finite-nucleus model, especially in the higher mass region. In Fig. 6 we compare our LDA single-neutron spectrum with that measured by the experiment KEK-E369 [6] in the case of ${ }_{\Lambda}^{89} \mathrm{Y}$. The decent description of data provided by the present LDA model for a hypernucleus as heavy as ${ }_{\Lambda}^{89} \mathrm{Y}$ is already an indicator of the reliability of the intranuclear cascade code used to simulate the nucleon FSI in hypernuclear decay.

\subsection{Ratio $N_{n} / N_{p}$}

We would like to compare now our results for the ratio between the number of neutrons and the number of protons produced in the decay of ${ }_{\Lambda}^{12} \mathrm{C}$ with experimental observations. Since our definitive aim is to determine $\Gamma_{n} / \Gamma_{p}$ by such a comparison, it is convenient [21] to start by introducing the number of nucleons of type $N(N=n$ or $p)$ produced, for a given energy cut, in $n-$ induced $\left(N_{N}^{1 \mathrm{Bn}}\right), p$-induced $\left(N_{N}^{1 \mathrm{Bp}}\right), n n$-induced $\left(N_{N}^{2 \mathrm{Bnn}}\right), n p$-induced $\left(N_{N}^{2 \mathrm{Bnp}}\right)$ and $p p$-induced $\left(N_{N}^{2 \mathrm{Bpp}}\right)$ decays. By normalizing these quantities per $n^{-}, p$-, 


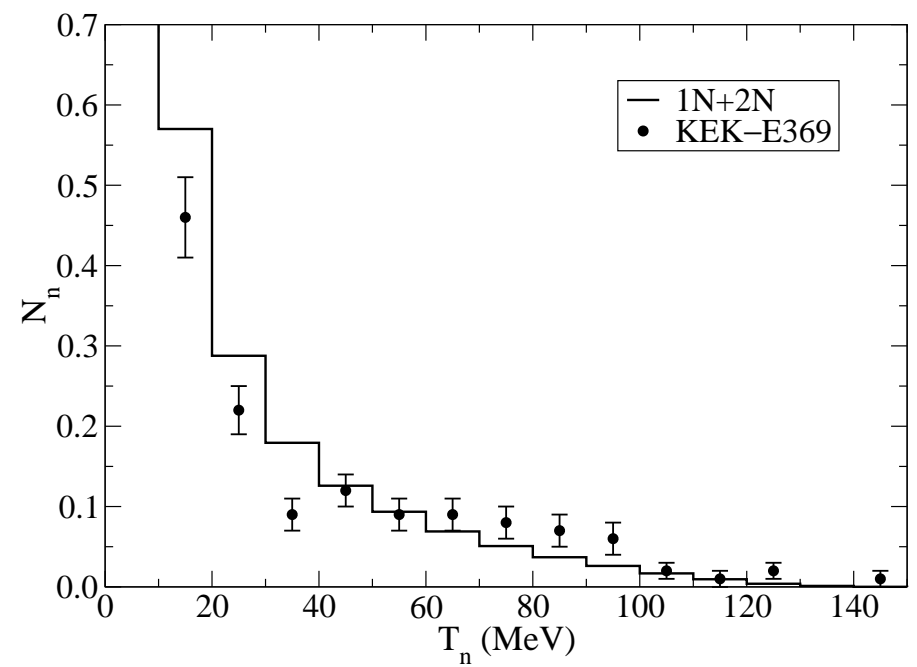

Fig. 6. Single-neutron kinetic energy spectra for the non-mesonic weak decay of ${ }_{\Lambda}^{89} \mathrm{Y}$. Data are from KEK-E369 [6]. All results are normalized per non-mesonic weak decay.

$n n-, n p-$ and $p p$-induced decay, respectively, the total number of nucleons of the type $N$ normalized per non-mesonic weak decay is given by:

$$
N_{N}=\frac{N_{N}^{1 \mathrm{Bn}} \Gamma_{n}+N_{N}^{1 \mathrm{Bp}} \Gamma_{p}+N_{N}^{2 \mathrm{Bnn}} \Gamma_{n n}+N_{N}^{2 \mathrm{Bnp}} \Gamma_{n p}+N_{N}^{2 \mathrm{Bpp}} \Gamma_{p p}}{\Gamma_{n}+\Gamma_{p}+\Gamma_{n n}+\Gamma_{n p}+\Gamma_{p p}} .
$$

By definition, the nucleon numbers $N_{N}^{1 \mathrm{Bn}}, N_{N}^{1 \mathrm{Bp}}$, etc, are independent of the model employed to describe the weak decay. In the absence of FSI effects, one would have $N_{n}^{1 \mathrm{Bn}}=2, N_{n}^{1 \mathrm{Bp}}=N_{p}^{1 \mathrm{Bp}}=1, N_{p}^{1 \mathrm{Bn}}=0, N_{n}^{2 \mathrm{Bnn}}=3, N_{p}^{2 \mathrm{Bnn}}=$ $0, N_{n}^{2 \mathrm{Bnp}}=N_{p}^{2 \mathrm{Bpp}}=2, N_{p}^{2 \mathrm{Bnp}}=N_{n}^{2 \mathrm{Bpp}}=1$. Their actual values depend on the strong interaction part of the problem, related to nucleon FSI, and on the framework (finite nucleus or nuclear matter) used for treating the hypernuclear structure effects, which produces different phase space factors. The dependence on the weak decay model enters Eq. (7) via the various partial decay widths.

In Table 2 we report our results for the weak decay model independent nucleon numbers for two kinetic energy thresholds for nucleon detection, $T_{N}^{\text {th }}=40$ and $60 \mathrm{MeV}$. From Eq. (7), our results of Table 1, and taking the values of Table 2 for $T_{N}^{\mathrm{th}}=60 \mathrm{MeV}$, we then determine:

$$
\frac{N_{n}}{N_{p}}=1.33
$$

We have to note that this result is close to the ones obtained in the finite nucleus calculation of [21], namely $N_{n} / N_{p}=1.38$ and 1.42 , using the OMEa 
Table 2

Predictions for the weak interaction model independent quantities $N_{N}^{1 \mathrm{Bn}}, N_{N}^{1 \mathrm{Bp}}$, $N_{N}^{2 \mathrm{Bnn}}, N_{N}^{2 \mathrm{Bnp}}, N_{N}^{2 \mathrm{Bpp}}$ and for $N_{N}^{2 \mathrm{~B}}$ of Eqs. (7) and (10)-(12) for ${ }_{\Lambda}^{12} \mathrm{C}$ by applying nucleon kinetic energy thresholds of $T_{N}^{\mathrm{th}}=40$ and $60 \mathrm{MeV}$.

\begin{tabular}{ccccccc}
\hline \hline$T_{N}^{\text {th }}(\mathrm{MeV})$ & $N_{n}^{1 \mathrm{Bn}}$ & $N_{n}^{1 \mathrm{Bp}}$ & $N_{n}^{2 \mathrm{Bnn}}$ & $N_{n}^{2 \mathrm{Bnp}}$ & $N_{n}^{2 \mathrm{Bpp}}$ & $N_{n}^{2 \mathrm{~B}}$ \\
\hline 40 & 1.26 & 0.71 & 1.10 & 0.80 & 0.35 & 0.73 \\
60 & 0.91 & 0.47 & 0.67 & 0.50 & 0.17 & 0.45
\end{tabular}

\begin{tabular}{ccccccc}
\hline \hline$T_{N}^{\text {th }}(\mathrm{MeV})$ & $N_{p}^{1 \mathrm{Bn}}$ & $N_{p}^{1 \mathrm{Bp}}$ & $N_{p}^{2 \mathrm{Bnn}}$ & $N_{p}^{2 \mathrm{Bnp}}$ & $N_{p}^{2 \mathrm{Bpp}}$ & $N_{p}^{2 \mathrm{~B}}$ \\
\hline 40 & 0.25 & 0.80 & 0.19 & 0.42 & 0.89 & 0.50 \\
60 & 0.11 & 0.54 & 0.08 & 0.21 & 0.56 & 0.27 \\
\hline \hline
\end{tabular}

and OMEf model, respectively. If the $2 N$-stimulated decay mode is neglected, the present calculation predicts

$$
\left(\frac{N_{n}}{N_{p}}\right)^{1 \mathrm{~N}}=1.28
$$

These results underestimate the value $N_{n} / N_{p}=2.00 \pm 0.17$ obtained by KEKE508 [7] for $T_{N}^{\text {th }}=60 \mathrm{MeV}$. Such an occurrence is related to the disagreement already observed for the single-proton spectra of Fig. 4. Our results for $T_{N}^{\mathrm{th}}=40 \mathrm{MeV}$, namely $N_{n} / N_{p}=1.26$ and $\left(N_{n} / N_{p}\right)^{1 \mathrm{~N}}=1.23$, do not compare well either with the previous experimental determination, $N_{n} / N_{p}=1.73 \pm 0.22$, obtained from KEK-E369 and KEK-E307 data [6] by applying the same detection threshold.

This discrepancy will obviously be reflected in the value of the $\Gamma_{n} / \Gamma_{p}$ ratio that can be extracted from the one-nucleon observables. From Eq. (7) one obtains:

$$
\frac{N_{n}}{N_{p}}=\frac{N_{n}^{1 \mathrm{Bn}} \frac{\Gamma_{n}}{\Gamma_{p}}+N_{n}^{1 \mathrm{Bp}}+\frac{1}{\Gamma_{1}}\left(1+\frac{\Gamma_{n}}{\Gamma_{p}}\right)\left(N_{n}^{2 \mathrm{Bnn}} \Gamma_{n n}+N_{n}^{2 \mathrm{Bnp}} \Gamma_{n p}+N_{n}^{2 \mathrm{Bpp}} \Gamma_{p p}\right)}{N_{p}^{1 \mathrm{Bn}} \frac{\Gamma_{n}}{\Gamma_{p}}+N_{p}^{1 \mathrm{Bp}}+\frac{1}{\Gamma_{1}}\left(1+\frac{\Gamma_{n}}{\Gamma_{p}}\right)\left(N_{p}^{2 \mathrm{Bnn}} \Gamma_{n n}+N_{p}^{2 \mathrm{Bnp}} \Gamma_{n p}+N_{p}^{2 \mathrm{Bpp}} \Gamma_{p p}\right)},
$$

from which one derives the ratio $\Gamma_{n} / \Gamma_{p}$ :

$$
\frac{\Gamma_{n}}{\Gamma_{p}}=\frac{N_{n}^{1 \mathrm{Bp}}+N_{n}^{2 \mathrm{~B}} \frac{\Gamma_{2}}{\Gamma_{1}}-\left(N_{p}^{1 \mathrm{Bp}}+N_{p}^{2 \mathrm{~B}} \frac{\Gamma_{2}}{\Gamma_{1}}\right) \frac{N_{n}}{N_{p}}}{\left(N_{p}^{1 \mathrm{Bn}}+N_{p}^{2 \mathrm{~B}} \frac{\Gamma_{2}}{\Gamma_{1}}\right) \frac{N_{n}}{N_{p}}-N_{n}^{1 \mathrm{Bn}}-N_{n}^{2 \mathrm{~B}} \frac{\Gamma_{2}}{\Gamma_{1}}}
$$


where

$$
N_{N}^{2 \mathrm{~B}} \equiv \frac{N_{N}^{2 \mathrm{Bnn}} \Gamma_{n n}+N_{N}^{2 \mathrm{Bnp}} \Gamma_{n p}+N_{N}^{2 \mathrm{Bpp}} \Gamma_{p p}}{\Gamma_{n n}+\Gamma_{n p}+\Gamma_{p p}} .
$$

Therefore, the ratio $\Gamma_{n} / \Gamma_{p}$ can be determined in terms of the observed ratio $N_{n} / N_{p}$, the weak-model independent nucleon numbers $N_{N}^{1 \mathrm{Bn}}, N_{N}^{1 \mathrm{Bp}}$, etc (which however depend on FSI effects), and a theoretical estimation for the twonucleon stimulated widths $\Gamma_{n n} / \Gamma_{1}, \Gamma_{n p} / \Gamma_{1}$ and $\Gamma_{p p} / \Gamma_{1}$. Using our predictions of Tables 1 and 2 together with the datum of KEK-E508 [7] for $T_{N}^{\text {th }}=60$ $\mathrm{MeV}, N_{n} / N_{p}=2.00 \pm 0.17$, we obtain:

$$
\frac{\Gamma_{n}}{\Gamma_{p}}=0.95 \pm 0.21
$$

Neglecting the $2 N$-stimulated channel the result is:

$$
\left(\frac{\Gamma_{n}}{\Gamma_{p}}\right)^{1 \mathrm{~N}}=0.88 \pm 0.16
$$

while enhancing arbitrarily the $2 N$-induced rates by a factor of two we obtain:

$$
\left(\frac{\Gamma_{n}}{\Gamma_{p}}\right)^{\Gamma_{2} \rightarrow 2 \Gamma_{2}}=1.02 \pm 0.27
$$

The sensitivity of the ratio $\Gamma_{n} / \Gamma_{p}$ to the values of the $2 N$-induced decay widths turns out to be moderate, i.e., well within the error bars. This is due not only to the minor role of the $2 N$-stimulated processes in Eq. (11) $\left(N_{n(p)}^{1 \mathrm{Bp}}\right.$ and $N_{n(p)}^{1 \mathrm{Bn}}$ are larger than $N_{n(p)}^{2 \mathrm{~B}} \Gamma_{2} / \Gamma_{1}$ since a quite high energy threshold is employed), but also to the particular value of $N_{n} / N_{p}$ used in the analysis, which causes a certain cancellation among the $2 N$-stimulated contributions in both the numerator and denominator of Eq. (11): $N_{n}^{2 \mathrm{~B}} \simeq N_{p}^{2 \mathrm{~B}} N_{n} / N_{p}$.

These occurrences can be better visualized in Fig. 7, which shows the relation between the observable ratio $N_{n} / N_{p}$ and $\Gamma_{n} / \Gamma_{p}$ for different choices of $\Gamma_{2} / \Gamma_{1}$ and assuming a detection threshold of $T_{N}=60 \mathrm{MeV}$. The dot-dashed line refers to the calculation in which $\Gamma_{2}$ is set to 0 , the continuous line to the $\Gamma_{2} / \Gamma_{1}$ ratio predicted by the present LDA model and the dashed line to the case in which the size of $\Gamma_{2}$ is arbitrarily doubled. The dotted line corresponds to the case in which $2 N$-induced decays and FSI are neglected. 


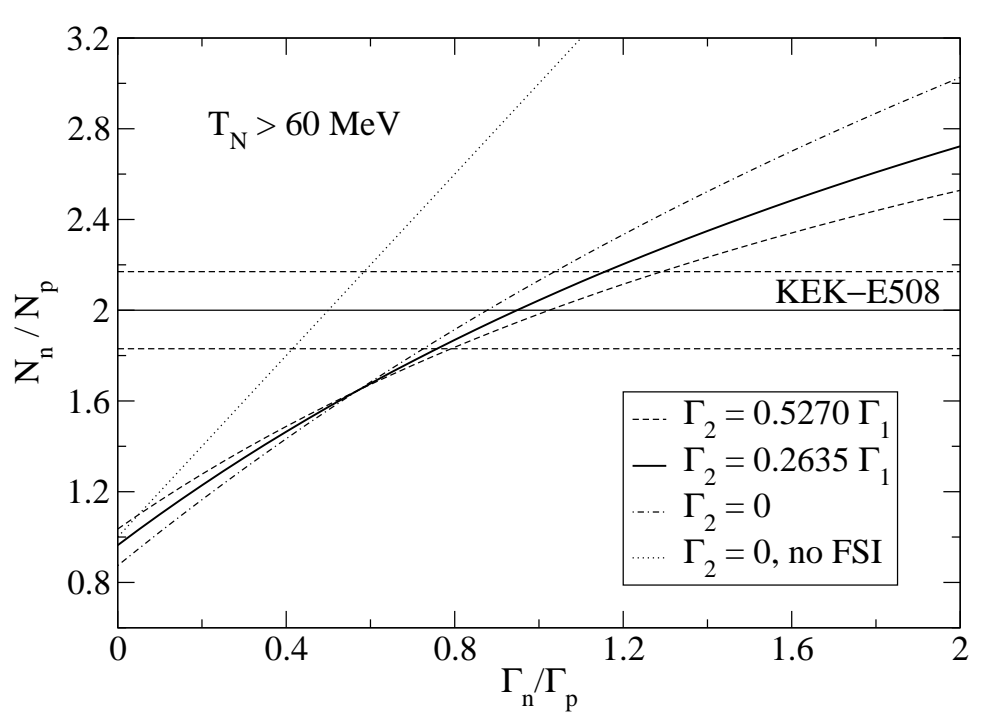

Fig. 7. Dependence of the observable ratio $N_{n} / N_{p}$ on $\Gamma_{n} / \Gamma_{p}$ and $\Gamma_{2} / \Gamma_{1}$ for ${ }_{\Lambda}^{12} \mathrm{C}$ and a nucleon energy threshold of $60 \mathrm{MeV}$. The horizontal lines show KEK-E508 data [7]. See text for further details.

Note that, even after applying a high kinetic thresholds such as $60 \mathrm{MeV}$, FSI effects are not negligible. This is why the previously extracted $\Gamma_{n} / \Gamma_{p}$ values are in disagreement with the value 0.5 given in [7] and expected on the basis of the relation $\Gamma_{n} / \Gamma_{p}=\left(N_{n} / N_{p}-1\right) / 2$, which holds if $2 N$-induced decays and FSI effects are ignored. On the contrary, it is well manifest from Fig. 7 that the $2 N$-induced decay mechanism plays a relatively small role in the whole range of reasonable $\Gamma_{n} / \Gamma_{p}$ values.

We end this subsection by remarking that the value of 0.285 for $\Gamma_{n} / \Gamma_{p}$ predicted by the weak decay model employed in the present paper, as well as those of the other available weak decay models, strongly underestimate the values of Eqs. (13)-(15). Again, this is just a consequence of the discrepancy found with the measured single-proton spectra, which in turn show an unfortunate dispersion among themselves. This, together with the strong effect of FSI depicted in Fig. 7 makes it advisable to resort to better suited quantities for the determination of $\Gamma_{n} / \Gamma_{p}$, such as the two-nucleon coincidence observables, in particular the ratio of $n n$ to $n p$ pairs discussed in Sect. 3.5.

\subsection{Double coincidence nucleon spectra}

Now we discuss the $N N$ coincidence spectra obtained from our model for the decay of ${ }_{\Lambda}^{12} \mathrm{C}$. Figs. 8 and 9 show, respectively, the distribution of $n n$ and $n p$ pairs, as a function of the cosine of the opening angle, where FSI effects have 


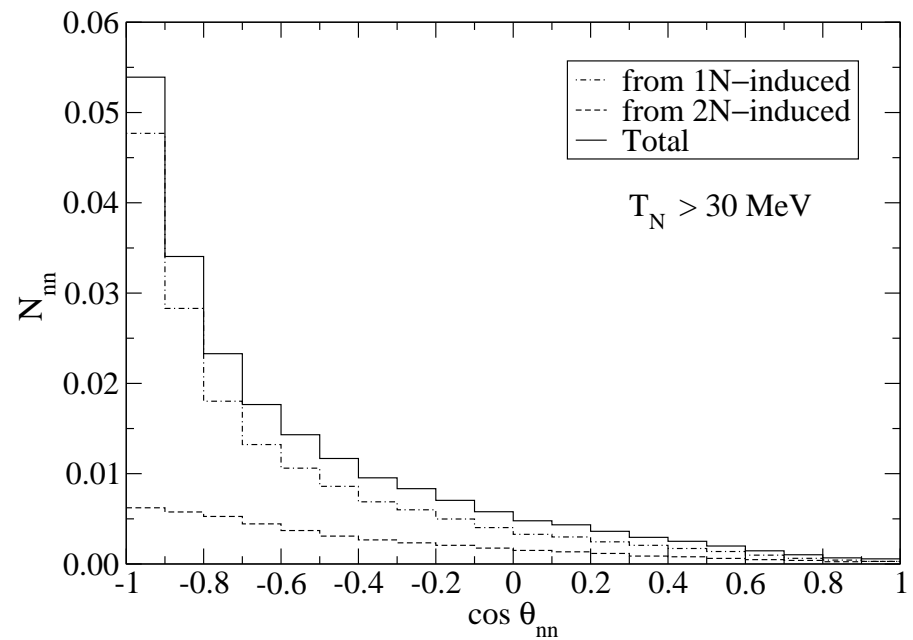

Fig. 8. Opening angle distribution of $n n$ pairs normalized per non-mesonic weak decay for ${ }_{\Lambda}^{12} \mathrm{C}$.

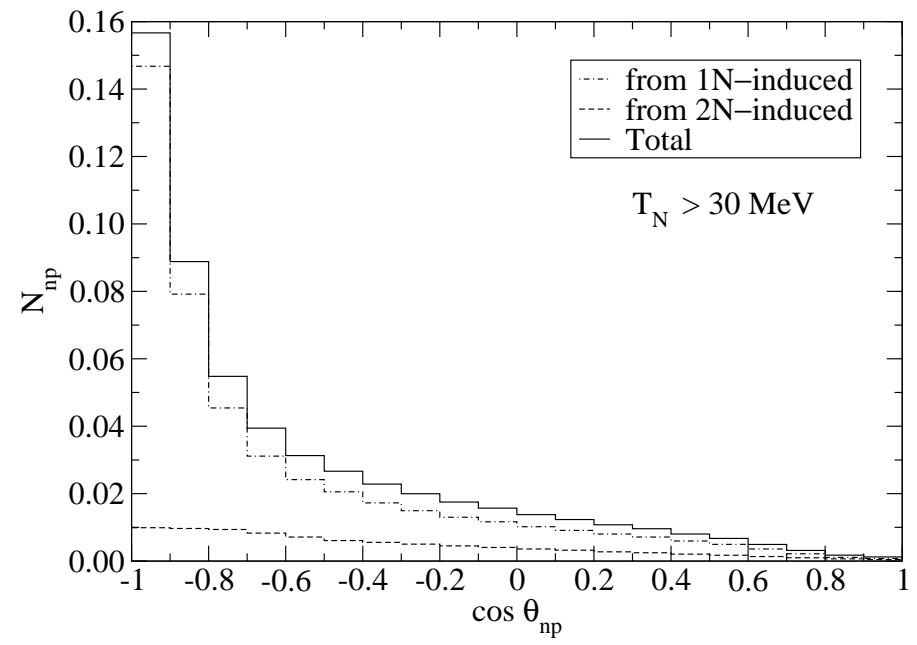

Fig. 9. Opening angle distribution of $n p$ pairs normalized per non-mesonic weak decay for ${ }_{\Lambda}^{12} \mathrm{C}$.

been incorporated and a kinetic energy cut of $30 \mathrm{MeV}$ has been applied. We observe that the distribution of pairs from the $1 N$-induced processes (dashdotted line) is more back-to-back dominated than the one coming from the $2 N$-induced processes (dashed line). In any case, the $1 N$-induced channel still provides the larger contribution of pairs in the whole range of opening angles.

For $\cos \theta_{N N} \lesssim-0.4$ the results of Figs. 8 and 9 reasonably reproduce the ones 
of the previous finite nucleus calculation reported in Fig. 5 of [20] and Fig. 9 of [21]. On the contrary, for $\cos \theta_{N N} \gtrsim-0.4$ a discrepancy is evident, the finite nucleus distributions being nearly flat in this region and the nuclear matter ones going monotonically to almost vanishing values with increasing values of $\cos \theta_{N N}$. This is just a reflection of the recoil effects present in a finite nucleus calculation, which make the $N N$ pairs to be less back-to-back correlated.

Our distributions of Figs. 8 and 9 can also be compared with those obtained by KEK-E508 and shown in Figure 3 of the paper by Kim et al. [10]. Due to the limited statistics of data, we concentrate on the angular region with $\cos \theta_{N N}<$ -0.7. In this region, the values $N_{n n}=0.083 \pm 0.014$ and $N_{n p}=0.138 \pm 0.014$ have been determined experimentally, whereas our corresponding results are $N_{n n}=0.111$ and $N_{n p}=0.300$. While there is decent agreement in the case of $N_{n n}$, we overestimate $N_{n p}$ by a factor of about 2 . This discrepancy is a consequence of the overestimation of the KEK-E508 single-proton spectra of Fig. 4. Another effect of the difference between the predicted and the measured number of protons can be seen when comparing our result for the number of proton-proton pairs for $T_{N}^{\mathrm{th}}=30 \mathrm{MeV}$ and $\cos \theta_{N N}<-0.7, N_{p p}=0.050$, with the experimental value $N_{p p}=0.005 \pm 0.002$.

The $n n$ and $n p$ pair distributions as functions of the pair kinetic energy are shown in Figs. 10 and 11, respectively, where the energy of each nucleon is larger than a threshold kinetic energy of $30 \mathrm{MeV}$. The upper panels show the distributions obtained without any cut in the opening angle, while in the bottom panels only the back-to-back events are kept by applying the restriction $\cos \theta_{N N}<-0.7$. We observe that the $2 N$-induced events (dashed lines) in the upper panels scarcely contribute at the position of the the primary peak of the $1 N$-induced contribution (dot-dashed lines). Instead, they enhance the total distribution at a pair energy of around $100 \mathrm{MeV}$, making the secondary peak (associated to the re-scattering events) there to become even higher (see the case of $n n$ pairs) than the primary one at the $Q$-value of about $155 \mathrm{MeV}$. When the angular cut is applied, many of the events in the low energy region are removed and the so-called back-to-back peak at $155 \mathrm{MeV}$ stands out more clearly, although there is still an important fraction of events that lie outside this peak. This is in quantitative agreement with the finite nucleus results of our previous works (see Fig. 3 of [20] and Fig. 10 of [21]), the only qualitative difference being the width of the back-to-back peak, which appears more smeared out in the present work due to Fermi motion. Note also that the distributions from $1 N$-induced decays of Figs. 10 and 11 extend above the largest possible $Q$-values, $Q \simeq 152 \mathrm{MeV}$ and $Q \simeq 157 \mathrm{MeV}$, corresponding to the three body non-mesonic decays ${ }_{\Lambda}^{12} \mathrm{C} \rightarrow{ }^{10} \mathrm{C}+n n$ and ${ }_{\Lambda}^{12} \mathrm{C} \rightarrow{ }^{10} \mathrm{~B}+n p$, 


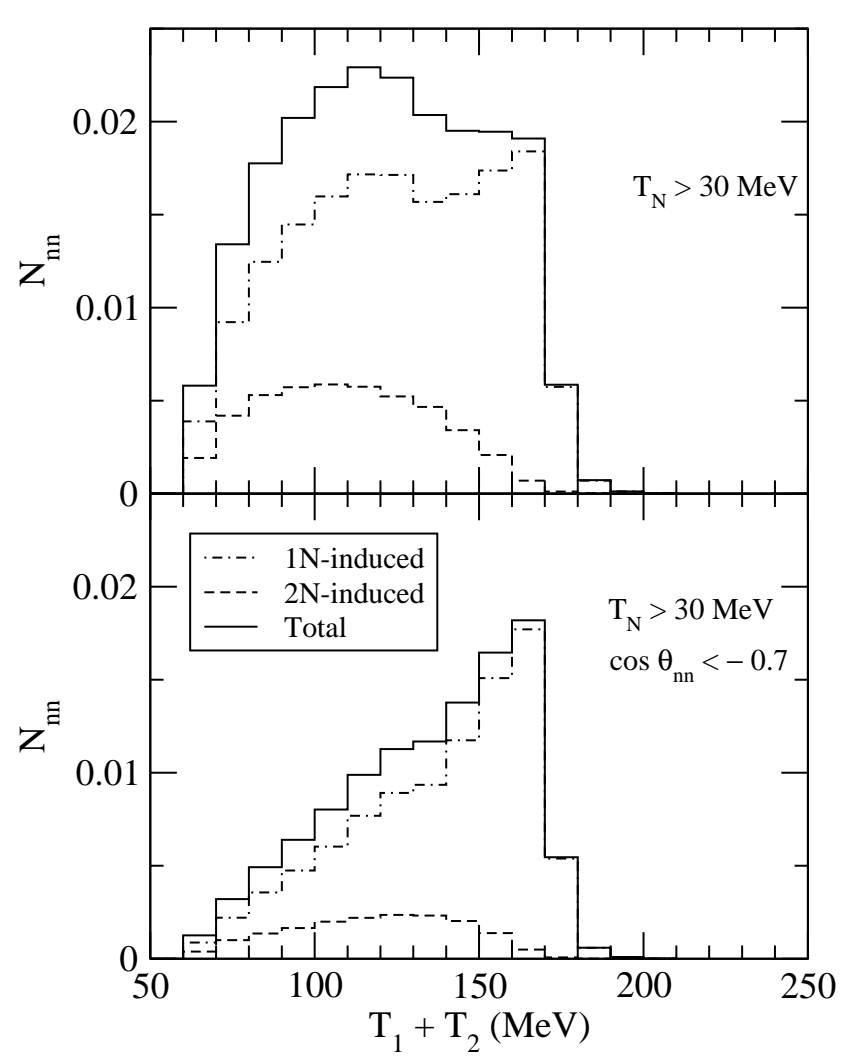

Fig. 10. Distribution of the total kinetic energy of $n n$ pairs normalized per non-mesonic weak decay for ${ }_{\Lambda}^{12} \mathrm{C}$. The energy threshold is $30 \mathrm{MeV}$ for each nucleon in the pair. In the upper panel there is no angular restriction, while the condition $\cos \theta_{n n}<-0.7$ has been imposed in the results of the lower panel.

respectively. These unwanted effects are typical of nuclear matter LDA-type calculations, which assume a continuum of states within the Fermi sea, and, therefore, cannot appropriately handle the discrete transition of the hypernuclear state to the ground state of the residual system, which should be signaled by a narrow peak in the distribution of nucleon pairs at a total kinetic energy equal to the $Q$-value of the reaction. This effect, however, does not affect the analysis nor the conclusions of the present work, which are based on the use of integrated quantities.

\section{$3.42 N-$ induced strength}

In this subsection we compare the results of our microscopic model for the $2 N$-induced channel with those of the phenomenological model of Ref. [44].

In Fig. 12 we compare the momentum distributions of each of the three primary nucleons emitted in $2 N$-induced processes (prior to FSI effects) for both 


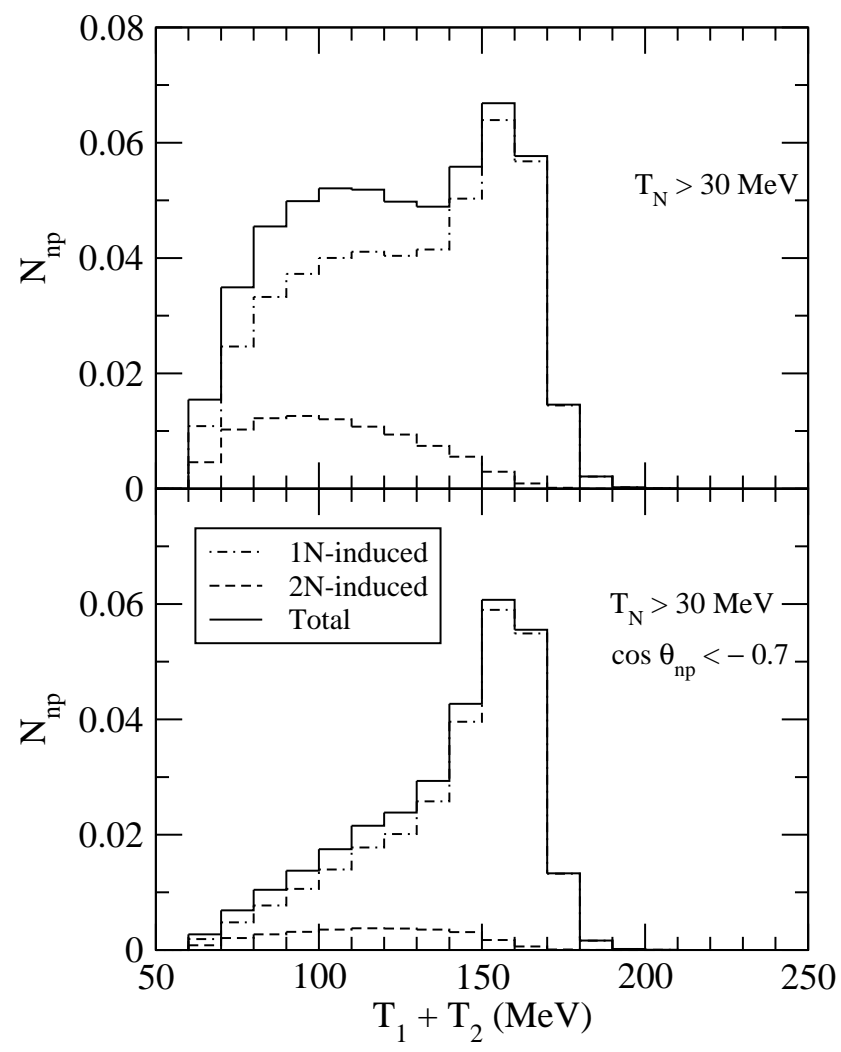

Fig. 11. Distribution of the total kinetic energy of $n p$ pairs normalized per non-mesonic weak decay for ${ }_{\Lambda}^{12} \mathrm{C}$. The energy threshold is $30 \mathrm{MeV}$ for each nucleon in the pair. In the upper panel there is no angular restriction, while the condition $\cos \theta_{n p}<-0.7$ has been imposed in the results of the lower panel.

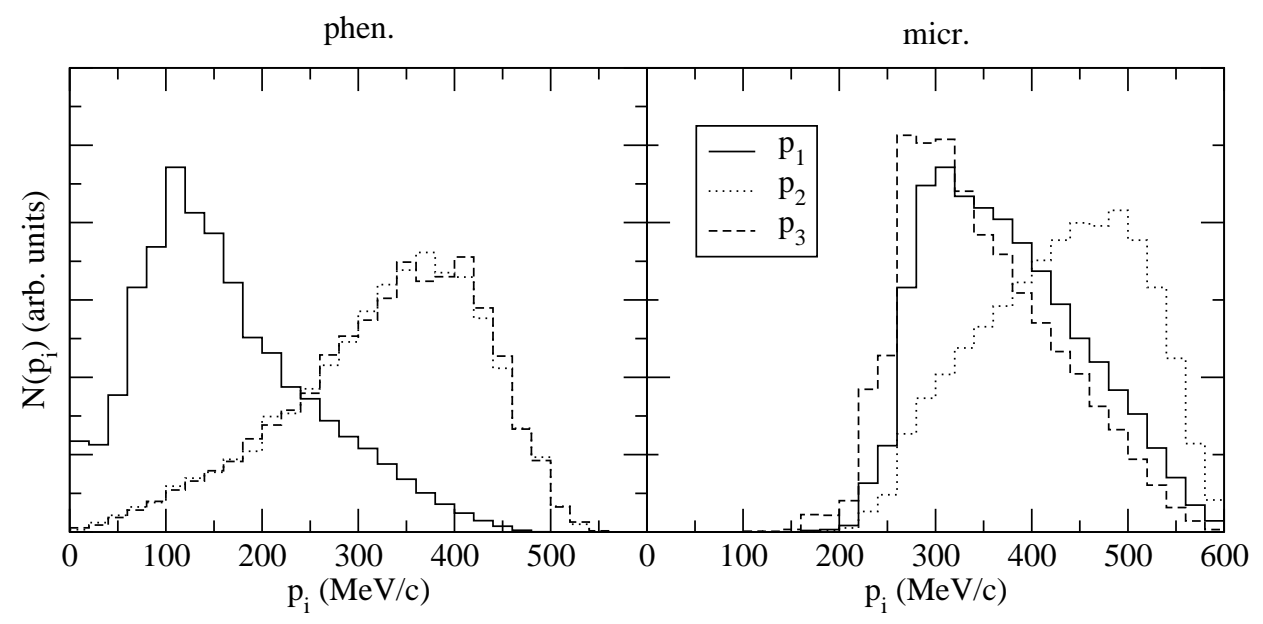

Fig. 12. Momentum distribution of each of the three primary nucleons emitted in $2 N$-induced decay processes for ${ }_{\Lambda}^{12} \mathrm{C}$.

models. The differences observed are a consequence of the particular dynamics embodied in each model. The phenomenological model assumes a one-pionexchange picture for the weak interaction and accounts for the phase space 
of $2 p 2 h$ excitations in the two-nucleon absorption process. Since the particular kinematical constraints of the $2 N$ induced channel allow for pions to be close to its mass-shell, this configuration will be especially enhanced. Consequently, the nucleon emitted at the $\Lambda N \pi$ vertex, whose momentum is denoted by $p_{1}$, has very little kinetic energy left, whereas the other two nucleons of the $2 N$-induced channel are quite energetic and come out in a practically backto-back geometry. In contrast, the microscopic one-meson-exchange model of Ref. [26] used in the present work weights each $2 p 2 h$ excitation with the strong interaction responsible for its coupling to the ground state. Therefore, the momentum $p_{2}$ of the nucleon emitted after absorbing the exchanged meson (see Fig. 2) can reach high values (close to $500 \mathrm{MeV} / \mathrm{c}$ ) from combining the momentum $q$ of the virtual meson with the momentum $p_{2}^{\prime}$ of the correlated nucleon, which can be large due to the hard nature of the short range $N N$ interaction. The momentum $p_{1}$, carried by the nucleon emitted at the $\Lambda$ vertex, and the momentum $p_{3}$, corresponding to the spectator nucleon, are quite similar, around $300 \mathrm{MeV} / \mathrm{c}$. This value is characteristic of the range of the interactions generating these nucleons, which are, respectively, the weak and strong one-meson-exchange models used in [26].

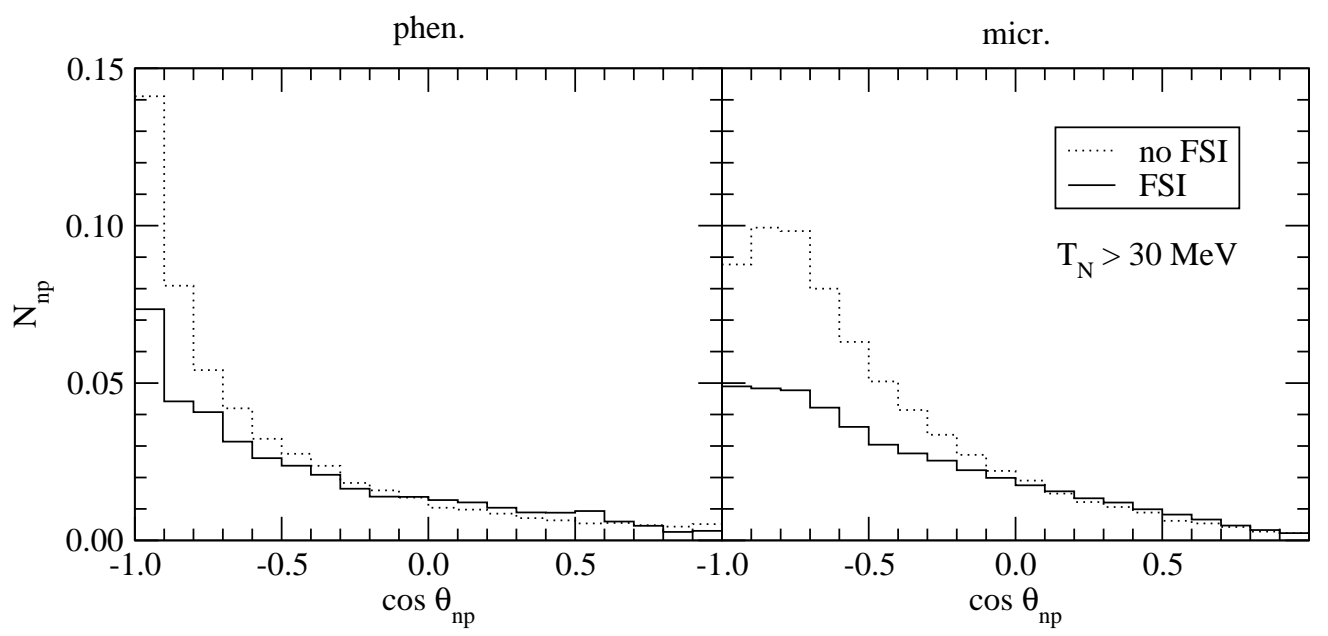

Fig. 13. Opening angle distribution of $n p$ pairs from the $n p$-induced channel for ${ }_{\Lambda}^{12} \mathrm{C}$, normalized per $n p$-induced decay.

We now compare the phenomenological and microscopic models after the three primary nucleons emitted in the weak decay process are allowed to undergo collisions with the other nucleons as they move out of the nucleus. We restrict here to the $n p$-induced decay mode, which is the most important one in the microscopic approach and the only one considered by the phenomenological model. The distribution of $n p$ pairs, normalized per its corresponding $n p$-induced transition rate, is shown in Fig. 13 as a function of the opening angle, where the threshold kinetic energy is $30 \mathrm{MeV}$ for each nucleon of the 
pair. The dotted (solid) line shows the results without (with) FSI effects. It is clear that, at variance to the microscopical approach, the phenomenological model produces a distinct back-to-back distribution which is partly kept even when FSI effects are included. The distribution of pairs as a function of the total kinetic energy and for a threshold $T_{N}^{\mathrm{th}}=30 \mathrm{MeV}$ is displayed in Fig. 14 . We observe that the amount of $N_{n p}$ pairs per $n p$-induced decay event in the absence of FSI (area under the dotted curves) is smaller in the phenomenological model, since the slow nucleon is always eliminated by the kinetic energy cut of $30 \mathrm{MeV}$. This situation is compensated when the opening angle cut is also applied (dashed lines), since it removes more events in the microscopic distribution, which is not so back-to-back dominated. Therefore, even if the kinematics of the primary nucleons look quite different, at the end, once the effect of FSI is considered and the energy and angular cuts are applied, both models produce similar neutron-proton angular and energy spectra per $n p$ induced decay event. This is a welcome feature since it will reduce the model dependence in the extraction of the $\Gamma_{n} / \Gamma_{p}$ ratio.

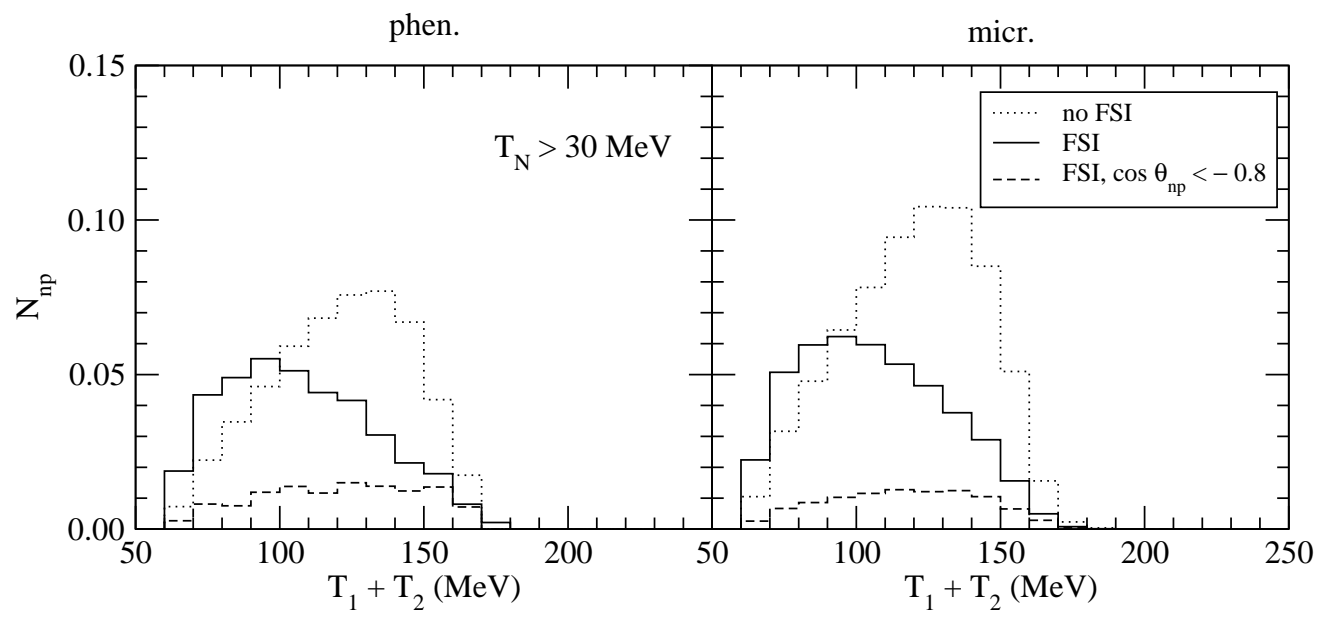

Fig. 14. Total kinetic energy distribution of $n p$ pairs from the $n p$-induced channel for ${ }_{\Lambda}^{12} \mathrm{C}$, normalized per $n p$-induced decay.

\subsection{Ratio $N_{n n} / N_{n p}$}

We start this section by comparing our predictions for the ratio between the number of $n n$ and $n p$ pairs, $N_{n n} / N_{n p}$, in ${ }_{\Lambda}^{12} \mathrm{C}$ with the experimental value obtained by the KEK-E508 experiment [8-10]. As in previous papers [20,21], we introduce the numbers of $N N$ pairs $(N N=n n, n p$ or $p p)$ coming from onenucleon induced $\left(N_{N N}^{1 \mathrm{Bn}}\right.$ and $\left.N_{N N}^{1 \mathrm{Bp}}\right)$ and two-nucleon induced $\left(N_{N N}^{2 \mathrm{Bnn}}, N_{N N}^{2 \mathrm{Bnp}}\right.$ and $N_{N N}^{2 \mathrm{Bpp}}$ ) processes, each one of them being normalized per the rate of the corresponding process. In the absence of FSI and ignoring the two-nucleon 
Table 3

Predictions for the weak interaction model independent quantities $N_{N N}^{1 \mathrm{Bn}}, N_{N N}^{1 \mathrm{Bp}}$, $N_{N N}^{2 \mathrm{Bnn}}, N_{N N}^{2 \mathrm{Bnp}}, N_{N N}^{2 \mathrm{Bpp}}$ and for $N_{N N}^{2 \mathrm{~B}}$ of Eqs. (16) and (17) for ${ }_{\Lambda}^{12} \mathrm{C}$, for nucleon energies $T_{N} \geq 30 \mathrm{MeV}$ and three angular regions.

\begin{tabular}{ccccccc}
\hline \hline & $N_{n n}^{1 \mathrm{Bn}}$ & $N_{n n}^{1 \mathrm{Bp}}$ & $N_{n n}^{2 \mathrm{Bnn}}$ & $N_{n n}^{2 \mathrm{Bnp}}$ & $N_{n n}^{2 \mathrm{Bpp}}$ & $N_{n n}^{2 \mathrm{~B}}$ \\
\hline all $\theta_{N N}$ & 0.55 & 0.11 & 0.53 & 0.24 & 0.05 & 0.22 \\
$\cos \theta_{N N}<-0.7$ & 0.37 & 0.05 & 0.21 & 0.09 & 0.01 & 0.08 \\
$\cos \theta_{N N}<-0.8$ & 0.31 & 0.03 & 0.15 & 0.06 & 0.01 & 0.06 \\
& & & & & & \\
& $N_{n p}^{1 \mathrm{Bn}}$ & $N_{n p}^{1 \mathrm{Bp}}$ & $N_{n p}^{2 \mathrm{Bnn}}$ & $N_{n p}^{2 \mathrm{Bnp}}$ & $N_{n p}^{2 \mathrm{Bpp}}$ & $N_{n p}^{2 \mathrm{~B}}$ \\
\hline $\operatorname{all} \theta_{N N}$ & 0.34 & 0.64 & 0.27 & 0.45 & 0.40 & 0.43 \\
$\cos \theta_{N N}<-0.7$ & 0.13 & 0.41 & 0.07 & 0.15 & 0.13 & 0.14 \\
$\cos \theta_{N N}<-0.8$ & 0.09 & 0.34 & 0.05 & 0.10 & 0.09 & 0.09 \\
& & & & & & \\
$\operatorname{all} \theta_{N N}$ & 0.04 & 0.15 & 0.02 & 0.08 & 0.30 & 0.12 \\
$\cos \theta_{N N}<-0.7$ & 0.01 & 0.06 & 0.005 & 0.02 & 0.12 & 0.04 \\
$\cos \theta_{N N}<-0.8$ & 0.01 & 0.05 & 0.004 & 0.02 & 0.08 & 0.03 \\
\hline \hline
\end{tabular}

induced channels, their values would be $N_{n n}^{1 \mathrm{Bn}}=N_{n p}^{1 \mathrm{Bp}}=1, N_{n p}^{1 \mathrm{Bn}}=N_{n n}^{1 \mathrm{Bp}}=0$ and $N_{N N}^{2 \mathrm{Bnn}}=N_{N N}^{2 \mathrm{Bnp}}=N_{N N}^{2 \mathrm{Bpp}}=0(N N=n n, n p$ or $p p)$. These weak decay model independent quantities, which are given in Table 3 for nucleon kinetic energies $T_{N} \geq 30 \mathrm{MeV}$ and three angular regions, determine the total number of $N N$ pairs emitted per non-mesonic weak decay event from:

$$
\begin{aligned}
N_{N N} & =\frac{N_{N N}^{1 \mathrm{Bn}} \Gamma_{n}+N_{N N}^{1 \mathrm{Bp}} \Gamma_{p}+N_{N N}^{2 \mathrm{Bnn}} \Gamma_{n n}+N_{N N}^{2 \mathrm{Bnp}} \Gamma_{n p}+N_{N N}^{2 \mathrm{Bpp}} \Gamma_{p p}}{\Gamma_{n}+\Gamma_{p}+\Gamma_{n n}+\Gamma_{n p}+\Gamma_{p p}} \\
& =\frac{N_{N N}^{1 \mathrm{Bn}} \Gamma_{n}+N_{N N}^{1 \mathrm{Bp}} \Gamma_{p}+N_{N N}^{2 \mathrm{~B}} \Gamma_{2}}{\Gamma_{n}+\Gamma_{p}+\Gamma_{2}}
\end{aligned}
$$

where

$$
N_{N N}^{2 \mathrm{~B}} \equiv \frac{N_{N N}^{2 \mathrm{Bnn}} \Gamma_{n n}+N_{N N}^{2 \mathrm{Bnp}} \Gamma_{n p}+N_{N N}^{2 \mathrm{Bpp}} \Gamma_{p p}}{\Gamma_{n n}+\Gamma_{n p}+\Gamma_{p p}} .
$$

Before proceeding with the discussion on the ratio $N_{n n} / N_{n p}$, we want to compare the results of Table 3 with the ones obtained within the finite nucleus framework of $[20,21]$ and reported in Table 4 . The agreement is in general 
Table 4

Predictions of the finite nucleus calculation of [21] for the weak interaction model independent quantities $N_{N N}^{1 \mathrm{Bn}}, N_{N N}^{1 \mathrm{Bp}}$ and for $N_{N N}^{2 \mathrm{~B}}$ (for nucleon energies $T_{N} \geq 30$ $\mathrm{MeV}$ and two angular regions) for ${ }_{\Lambda}^{12} \mathrm{C}$.

\begin{tabular}{cccc}
\hline \hline & $N_{n n}^{1 \mathrm{Bn}}$ & $N_{n n}^{1 \mathrm{Bp}}$ & $N_{n n}^{2 \mathrm{~B}}$ \\
\hline all $\theta_{N N}$ & 0.57 & 0.11 & 0.30 \\
$\cos \theta_{N N}<-0.8$ & 0.31 & 0.03 & 0.12 \\
& & & \\
& $N_{n p}^{1 \mathrm{Bn}}$ & $N_{n p}^{1 \mathrm{Bp}}$ & $N_{n p}^{2 \mathrm{~B}}$ \\
\hline all $\theta_{N N}$ & 0.34 & 0.68 & 0.39 \\
$\cos \theta_{N N}<-0.8$ & 0.09 & 0.32 & 0.10 \\
& & & \\
\hline $\operatorname{all} \theta_{N N}$ & 0.04 & 0.17 & 0.05 \\
$\cos \theta_{N N}<-0.8$ & 0.01 & 0.05 & 0.01 \\
\hline \hline
\end{tabular}

very good, especially in the case of the $1 N$-induced contributions. This is expected since these numbers are independent of the weak decay model and, in addition, the FSI effects are modeled with the same intranuclear cascade code in both evaluations. Some discrepancies are observed in a few $2 N$-induced contributions. The reason is that the different primary nucleon momentum distributions determined by the two-nucleon absorption models (see Fig. 12) are influenced differently by FSI effects. The significance of these discrepancies is anyhow relatively low for the total numbers $N_{N N}$ due to the smallness of the corresponding decay rates.

We now come back to the $N_{n n} / N_{n p}$ ratio. From Eq. (16) and our results of Tables 1 and 3 we obtain:

$$
\frac{N_{n n}}{N_{n p}}=0.37(0.36)
$$

for the case with $\cos \theta_{N N} \leq-0.7$ (in parentheses we give the predictions for $\left.\cos \theta_{N N} \leq-0.8\right)$. The result for $\cos \theta_{N N} \leq-0.8$ is in agreement with the KEK-E508 experiment, for which $N_{n n} / N_{n p}=0.40 \pm 0.10$, but the prediction for $\cos \theta_{N N} \leq-0.7$ underestimates the datum from the same experiment, $0.60 \pm 0.12$, which is the one used in Ref. [10] to determine $\Gamma_{n} / \Gamma_{p}$. Clearly, the theoretical predictions for $N_{n n} / N_{n p}$ are less dependent than the data for variations of the opening angle region. The differences among our result of 
Eq. (18) for $\cos \theta_{N N} \leq-0.8$ and the finite nucleus predictions of [21] (from Tables 1 and 4), namely $N_{n n} / N_{n p}=0.43$ and 0.47 for the OMEa and OMEf models, respectively, are due to the different partial decay rates and phase spaces predicted by the different models. If the $2 N$-stimulated decay mode is neglected, we obtain:

$$
\left(\frac{N_{n n}}{N_{n p}}\right)^{1 \mathrm{~N}}=0.35(0.34)
$$

for $\cos \theta_{N N} \leq-0.7\left(\cos \theta_{N N} \leq-0.8\right)$, thus emphasizing a relatively small effect of the $2 N$-induced channels on correlation observables appropriately chosen. These last results are not so different from the naïve estimation $N_{n n} / N_{n p}=$ $\Gamma_{n} / \Gamma_{p}=0.285$ obtained when FSI are also ignored, supporting the claim that FSI effects in the case of coincidence observables is limited and, in fact, they can be narrowed further down by using higher kinetic energy thresholds thresholds and more restrictive back-to-back constraints. This gives us confidence that the ratio $N_{n n} / N_{n p}$ is in fact a much more trustable and better controlled observable to determine $\Gamma_{n} / \Gamma_{p}$.

We now attempt to extract the ratio $\Gamma_{n} / \Gamma_{p}$ from a weak decay model independent analysis of the experimental double-coincidence data for $N_{n n} / N_{n p}$, and using our theoretical estimates for $\Gamma_{n n} / \Gamma_{1}, \Gamma_{n p} / \Gamma_{1}$ and $\Gamma_{p p} / \Gamma_{1}$. From Eqs. (16) and (17), applied to $N N=n n$ and $n p$, one obtains:

$$
\frac{\Gamma_{n}}{\Gamma_{p}}=\frac{N_{n n}^{1 \mathrm{Bp}}+N_{n n}^{2 \mathrm{~B}} \frac{\Gamma_{2}}{\Gamma_{1}}-\left(N_{n p}^{1 \mathrm{Bp}}+N_{n p}^{2 \mathrm{~B}} \frac{\Gamma_{2}}{\Gamma_{1}}\right) \frac{N_{n n}}{N_{n p}}}{\left(N_{n p}^{1 \mathrm{Bn}}+N_{n p}^{2 \mathrm{~B}} \frac{\Gamma_{2}}{\Gamma_{1}}\right) \frac{N_{n n}}{N_{n p}}-N_{n n}^{1 \mathrm{Bn}}-N_{n n}^{2 \mathrm{~B}} \frac{\Gamma_{2}}{\Gamma_{1}}} .
$$

For the ${ }_{\Lambda}^{12} \mathrm{C}$ datum of $\mathrm{KEK}-\mathrm{E} 508, N_{n n} / N_{n p}=0.60 \pm 0.12$, corresponding to the cuts $T_{N} \geq 30 \mathrm{MeV}$ and $\cos \theta_{N N} \leq-0.7$, we derive:

$$
\frac{\Gamma_{n}}{\Gamma_{p}}=0.66 \pm 0.24
$$

which agrees with the determination of KEK-E508: $\left(\Gamma_{n} / \Gamma_{p}\right)^{\exp }=0.51 \pm 0.13 \pm$ 0.05 [10]. By neglecting the $2 N$-stimulated channel we obtain:

$$
\left(\frac{\Gamma_{n}}{\Gamma_{p}}\right)^{1 \mathrm{~N}}=0.66 \pm 0.21
$$


whereas enhancing arbitrarily the $2 N$-induced rates by a factor of two we find:

$$
\left(\frac{\Gamma_{n}}{\Gamma_{p}}\right)^{\Gamma_{2} \rightarrow 2 \Gamma_{2}}=0.67 \pm 0.27
$$

Note that the above $\Gamma_{n} / \Gamma_{p}$ ratios are much larger than the values extracted in Ref. [21]: for instance, the value of Ref. [21] corresponding to Eq. (21) is $\Gamma_{n} / \Gamma_{p}=0.27 \pm 0.14$. This apparent disagreement is only an effect of the different data used in the two fits: the present determinations are based on the datum $\left(N_{n n} / N_{n p}\right)^{\exp }\left(\cos \theta_{N N} \leq-0.7\right)=0.60 \pm 0.12$, while the ones of Ref. [21] used $\left(N_{n n} / N_{n p}\right)^{\exp }\left(\cos \theta_{N N} \leq-0.8\right)=0.40 \pm 0.10$. A fit performed with the present framework and adopting the datum for $\cos \theta_{N N} \leq-0.8$ would provide a value for $\Gamma_{n} / \Gamma_{p}=0.34 \pm 0.15$, in good agreement with the determinations of Ref. [21]. This indicates a moderate model dependence in the extraction of the $\Gamma_{n} / \Gamma_{p}$ ratio from double--coincidence observables.

Another, more illustrative, way of visualizing the dependence of the $\Gamma_{n} / \Gamma_{p}$ ratio on the value of $\Gamma_{2}$, as well as on FSI effects, is displayed in Fig. 15, where the relation between $N_{n n} / N_{n p}$ and $\Gamma_{n} / \Gamma_{p}$ is shown for different choices of $\Gamma_{2} / \Gamma_{1}$ and for $T_{N}^{\mathrm{th}}=30 \mathrm{MeV}$ and $\cos \theta_{N N} \leq-0.7$. The dotted line corresponds to the case in which $2 \mathrm{~N}$-induced decays and FSI are neglected. The other three lines incorporate FSI and different choices for $\Gamma_{2}$ : a vanishing value (dotdashed line), the two-nucleon induced rates $\Gamma_{n p}=0.138, \Gamma_{p p}=0.036$, and $\Gamma_{n n}=0.010$ predicted by the present LDA model (continuous line) and a case in which these rates are arbitrarily doubled (dashed line). The figure clearly shows the very small effect of $\Gamma_{2}$ in the determination of $\Gamma_{n} / \Gamma_{p}$ from the measured $N_{n n} / N_{n p}$. One can also see, upon comparing Fig. 15 with Fig. 7 , that FSI affect much more the value of $\Gamma_{n} / \Gamma_{p}$ extracted from a the singlenucleon observable $N_{n} / N_{p}$.

\section{Conclusions}

We have presented a study of single- and double-coincidence nucleon spectra for the non-mesonic weak decay of $\Lambda$-hypernuclei, within a nuclear matter framework which has been adapted to finite nuclei via the local density approximation. One-meson-exchange models have been used to describe oneand two-nucleon induced decay processes, $\Lambda N \rightarrow n N$ and $\Lambda N N \rightarrow n N N$. Final state interactions of the outgoing nucleons with the residual nucleus have been simulated with an intranuclear cascade code based on Monte Carlo 


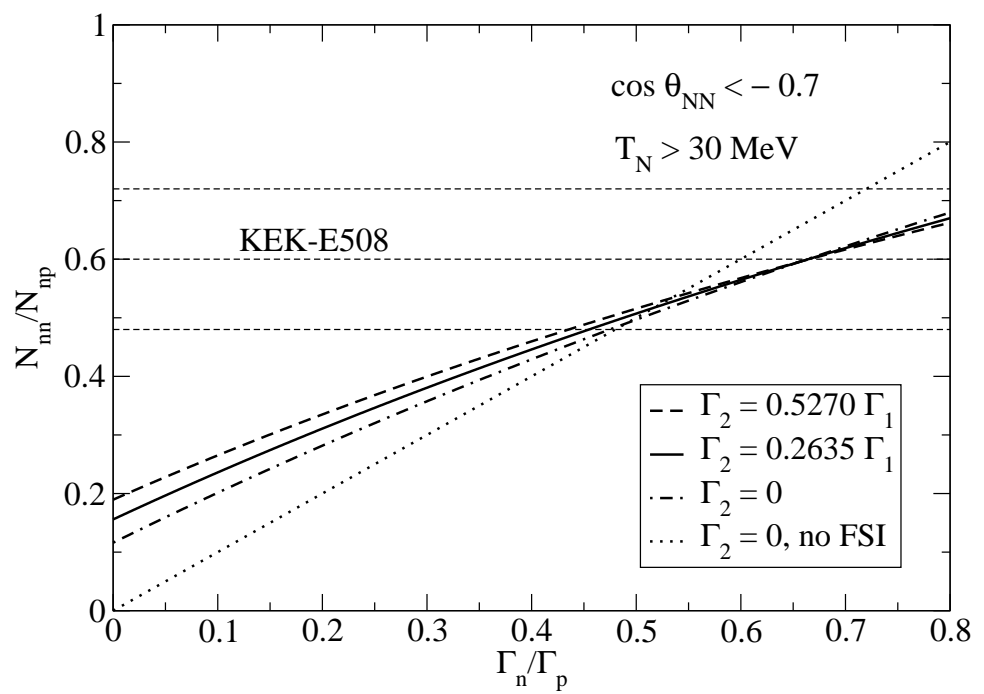

Fig. 15. Dependence of the observable ratio $N_{n} / N_{p}$ on $\Gamma_{n} / \Gamma_{p}$ and $\Gamma_{2} / \Gamma_{1}$ for ${ }_{\Lambda}^{12} \mathrm{C}$, for a nucleon energy threshold of $30 \mathrm{MeV}$ and $\cos \theta_{N N} \leq-0.7$. The horizontal lines show KEK-E508 data [10]. See text for further details.

techniques.

Unlike previous papers, we have adopted here a microscopic approach to the two-nucleon induced decay mode, which includes the isospin channels $\Lambda n n \rightarrow$ $n n n$ and $\Lambda p p \rightarrow n p p$, in addition to the dominant $\Lambda n p \rightarrow n n p$ mode considered in the phenomenological studies. The consideration of a different model for the two-nucleon induced rate is also helpful for establishing the amount of model dependency of the extracted value of the ratio $\Gamma_{n} / \Gamma_{p}$ from data.

Our results have been compared with previous finite nucleus analysis [20, 21]. Apart from some differences ascribable to the phase space dependence and to the different models for the two-nucleon induced decay channels, the present predictions for the observable ratios $N_{n} / N_{p}$ and $N_{n n} / N_{n p}$ confirm the finite nucleus results.

There is a considerable amount of data $[5-10,15,17,38,40,41]$ to which our results can be compared. The single-neutron spectra for ${ }_{\Lambda}^{12} \mathrm{C}$ and ${ }_{\Lambda}^{89} \mathrm{Y}$ measured by KEK-E369 [6] and KEK-E508 [8] are satisfactorily reproduced. Our prediction for the ${ }_{\Lambda}^{12} \mathrm{C}$ single-proton spectrum overestimates the one measured by the KEK-E508 experiment [8], although the shape is reasonably reproduced. Our spectrum does not show the shallow peak at $T_{p} \simeq 80 \mathrm{MeV}$ reported recently by the FINUDA collaboration $[15,17]$. We note, however, that there exists a certain dispersion between the different measured proton spectra, which we hope will be resolved by the future experiments planned at Daphne, J-PARC and HypHI@FAIR. 
From the single-nucleon observable $N_{n} / N_{p}$, we have determined the value of the $\Gamma_{n} / \Gamma_{p}$ ratio, analyzing in detail the effect of the two-nucleon stimulated decay branches as well as that of FSI. The result obtained, $\Gamma_{n} / \Gamma_{p}=0.95 \pm 0.21$, is clearly larger than pure theoretical predictions, which range between 0.3 and 0.5. However, while the contribution of the two-nucleon induced decay channels in this analysis turns out to be of moderate size, FSI effects appear to be of great importance.

Nucleon-nucleon correlation observables can be much less affected by FSI, as we have explicitly shown here, especially when appropriate cuts are applied. Comparing our predictions of nucleon-nucleon pairs with KEK-E508 data [10], we find a fair agreement for $N_{n n}$, while we overestimate the observations for $N_{n p}$ and $N_{p p}$, which is a similar type of discrepancy as that observed for the single-proton spectra.

We have determined $\Gamma_{n} / \Gamma_{p}$ from the experimental value of the $N_{n n} / N_{n p}$ ratio, using a a weak model independent analysis, as done also in previous works $[20,21]$, but employing here the microscopic estimation of the two-nucleon stimulated processes. The KEK $N_{n n} / N_{n p}$ datum for ${ }_{\Lambda}^{12} \mathrm{C}$, a nucleon kinetic energy threshold of $30 \mathrm{MeV}$ and an opening angle range $\cos \theta_{N N} \leq-0.7$ is reproduced with $\Gamma_{n} / \Gamma_{p}=0.66 \pm 0.24$. A very small dependence on the twonucleon stimulated channels is found for the extracted ratio. The fitted value of $\Gamma_{n} / \Gamma_{p}$ is in agreement with the KEK determination, $0.51 \pm 0.13 \pm 0.05$ [10], and with some of today's pure theoretical evaluations - which also suffer from a certain degree of arbitrariness in the experimentally unknown baryon couplings - ranging in the interval 0.3-0.5.

To summarize, while we can assert that analyses of correlation measurements produce values for the ratio $\Gamma_{n} / \Gamma_{p}$ in agreement with theoretical predictions, single-nucleon spectra are less trustable, not only because their analysis is more affected by FSI effects, but also because the experimental results still show a certain amount of dispersion. It is our hope that the data expected for the near future from Daphne, J-PARC and HypHI will help in clarifying the situation. We finally note that the present work should be seen as the starting point for more complex calculations. Additional diagrams contributing to $\Gamma_{2}$ and including both direct and exchange terms, ground state correlations contributions to $\Gamma_{1}$, quantum-mechanical formulations (alternative to the INC) for the FSI taking care of the quantum interference terms, etc., are all topics which can be important $[27,28]$ and have not been incorporated yet in the present problem. Due to the huge amount of configurations involved, an implementation of these effects should be first accomplished in nuclear matter. The 
agreement and consistency between the finite nucleus results of Refs. [20,21] with those found in this work within a nuclear matter approach, makes it advisable to attempt this goal using this last scheme.

\section{Acknowledgments}

This work is partly supported by the EU contract No. MRTN-CT-2006-035482 (FLAVIAnet), by the contract FIS2008-01661 from MICINN (Spain), by the Generalitat de Catalunya contract 2009SGR-1289, by INFN-MEC collaboration agreement number 06-36, and by CONICET (Argentina) under contract PIP 6159. We acknowledge the support of the European Community-Research

Infrastructure Integrating Activity "Study of Strongly Interacting Matter" (HadronPhysics2, Grant Agreement n. 227431) under the Seventh Framework Programme of EU.

\section{References}

[1] E. Oset and A. Ramos, Prog. Part. Nucl. Phys. 41, 191-253 (1998).

[2] W. M. Alberico and G. Garbarino, Phys. Rep. 369, 1 (2002); in Hadron Physics, IOS Press, Amsterdam, 2005, p. 125. Edited by T. Bressani, A. Filippi and U. Wiedner. Proceedings of the International School of Physics "Enrico Fermi", Course CLVIII, Varenna (Italy), June 22 - July 2, 2004.

[3] A. Parreño, Lect. Notes Phys. 724, 141 (2007).

[4] O. Hashimoto et al., Phys. Rev. Lett. 88, 042503 (2002).

[5] Y. Sato et al., Phys. Rev. C 71, 025203 (2005).

[6] J. H. Kim el al., Phys. Rev. C 68, 065201 (2003).

[7] S. Okada et al., Phys. Lett. B 597, 249 (2004).

[8] H. Outa, in Hadron Physics (see [2]) p. 219; H. Outa et al., Nucl. Phys. A 754, 157c (2005).

[9] B. H. Kang et al., Phys. Rev. Lett. 96, 062301 (2006).

[10] M. J. Kim et al., Phys. Lett. B 641, 28 (2006).

[11] H. Bhang et al., Eur. Phys. J. A 33, 259 (2007).

[12] M. Kim et al., Phys. Rev. Lett. 103, 182502 (2009).

[13] J.D. Parker et al., Phys. Rev. C 76, 035501 (2007). 
[14] M. Agnello et al., Phys. Lett. B 622, 35 (2005).

[15] M. Agnello et al., Nucl. Phys. A 804, 151 (2008).

[16] M. Agnello et al., Phys. Lett. B 681, 139 (2009).

[17] M. Agnello et al., e-Print: arXiv:0910.4939 [nucl-ex].

[18] T. Nagae, Nucl. Phys. A 754, 443c (2005); Nucl.Phys. A 805, 486c (2008).

[19] T. Fukuda et al., Nucl. Phys. A 790, 161c (2007).

[20] G. Garbarino, A. Parreño and A. Ramos, Phys. Rev. Lett. 91, 112501 (2003).

[21] G. Garbarino, A. Parreño and A. Ramos, Phys. Rev. C 69, 054603 (2004); W. M. Alberico, G. Garbarino, A. Parreño and A. Ramos, nucl-th/0407046, in DAPHNE 2004: Physics at meson factories, Frascati Phys. Ser. 36, 249 (2005). Edited by F. Anulli, M. Bertani, G. Capon, C. Curceanu-Petrascu, F. L. Fabbri and S. Miscetti.

[22] D. Jido, E. Oset and J. E. Palomar, Nucl. Phys. A 694, 525 (2001).

[23] A. Parreño and A. Ramos, Phys. Rev. C 65, 015204 (2002); A. Parreño, A. Ramos and C. Bennhold, Phys. Rev. C 56, 339 (1997).

[24] K. Itonaga, T. Ueda and T. Motoba, Phys. Rev. C 65, 034617 (2002).

[25] E. Bauer and F. Krmpotić, Nucl. Phys. A 717, 217 (2003).

[26] E. Bauer and F. Krmpotić, Nucl. Phys. A 739, 109 (2004).

[27] E. Bauer, Nucl. Phys. A 818, 174 (2009).

[28] E. Bauer and G. Garbarino, Nucl. Phys. A 828, 29 (2009).

[29] T. Maruta et al., PhD thesis, KEK Report 2006-1, June 2006; in Proceedings of the IX International Conference on Hypernuclear and Strange Particle Physics, edited by J. Pochodzalla and Th. Walcher (SIF and Springer, Berlin Heidelberg, 2007) p.97.

[30] W.M. Alberico, G. Garbarino, A. Parreño and A. Ramos, Phys. Rev. Lett. 94, 082501 (2005).

[31] C. Barbero, C. De Conti, A. P. Galeão and F. Krmpotić, Nucl. Phys. A 726, 267 (2003); C. Barbero, A. P. Galeão and F. Krmpotić, Phys. Rev. C 72, 035210 (2005).

[32] K. Sasaki, T. Inoue and M. Oka, Nucl. Phys. A 707, 477 (2002).

[33] A. Parreño, C. Bennhold and B.R. Holstein, Phys. Rev. C 70, 051601(R) (2004); Nucl. Phys. A 754, 127c (2005).

[34] K. Itonaga, T. Motoba and T. Ueda, in Electrophoto-Production of Strangeness on Nucleons and Nuclei, edited by K. Maeda, H. Tamura, S. N. Nakamura and O. Hashimoto (World Scientific, Singapore, 2004) p. 397; 
[35] K. Sasaki, M. Izaki, and M. Oka, Phys. Rev. C 71, 035502 (2005).

[36] C. Barbero and A. Mariano, Phys. Rev. C 73, 024309 (2006).

[37] C. Chumillas, G. Garbarino, A. Parreno and A. Ramos, Phys. Lett. B 657, 180 (2007).

[38] A. Montwill et al., Nucl. Phys. A 234, 413 (1974).

[39] A. Sakaguchi et al., Phys. Rev. C 43, 73 (1991).

[40] J. J. Szymanski et al., Phys. Rev. C 43, 849 (1991).

[41] H. Noumi et al., Phys. Rev. C 52, 2936 (1995).

[42] H. Noumi et al., Proceedings of the IV International Symposium on Weak and Electromagnetic Interactions in Nuclei, edited by H. Ejiri, T. Kishimoto and T. Sato (World Scientific, Singapore, 1995), p. 550.

[43] A. Ramos, M. J. Vicente-Vacas and E. Oset, Phys. Rev. C 55, 735 (1997); 66, 039903(E) (2002).

[44] A. Ramos, E. Oset and L. L. Salcedo, Phys. Rev. C 50, 2314 (1994).

[45] E. Oset and L. L. Salcedo, Nucl. Phys. A 443, 704 (1985).

[46] W. M. Alberico, A. De Pace, M. Ericson and A. Molinari, Phys. Lett. B 256, 134 (1991).

[47] R. Machleidt, K. Holinde and Ch. Elster; Phys. Rep. 149, 1 (1987).

[48] M. B. Barbaro, A. De Pace, T. W. Donnelly and A. Molinari, Nucl. Phys. A 596, 553 (1996).

[49] L. L. Salcedo, E. Oset, M. J. Vicente-Vacas and C. Garcia-Recio, Nucl. Phys. A 484, 557 (1988); R. C. Carrasco, M. J. Vicente-Vacas and E. Oset, Nucl. Phys. A 570, 701 (1994); A. Gil, J. Nieves and E. Oset, Nucl. Phys. A 627, 599 (1997).

[50] V. G. J. Stoks and T. A. Rijken, Phys. Rev. C 59, 3009 (1999). 